\title{
A Twitter Framework to assess the Effectiveness of Indian Government Campaign
}

\author{
AARZOO DHIMAN, Indian Institute of Technology Roorkee, India \\ DURGA TOSHNIWAL, Indian Institute of Technology Roorkee, India
}

Many government and private agencies introduce social, governmental, educational, commercial and public-health based
campaigns to refine various sections of the society. Traditionally, citizen feedback and on-field surveys are performed to
assess the effectiveness of such campaigns. However, there is limited availability of the social media tools that assess the
impact of different government campaigns automatically. In this research work, a framework has been proposed which uses
the social media data i.e. Twitter data pertaining to an Indian Cleanliness Campaign Swachh Bharat Abhiyan (SBA) to perform
the effectiveness assessment. This research work has been performed in two parts. First, Twitter data has been processed to
predict the perceptions of citizens using Word Embeddings-based Tweet Pooling and Subjectivity score-based Sentiment Analysis
and second, the performance of the cities has been predicted using the demographic features based predictor models. The
experimentation shows a 0.77 correlation between the proposed framework and the government surveys while predicting the
citizensâǍ́z perspectives and $80(+/-15) \%$ accuracy while predicting the performance of cities using Random Forest Regression.
Furthermore, the cities have been clustered using Twitter and the demographic data to find out the interesting patterns and
behaviors. This research work provides better insights to the potential of social media data in the interventional studies of the
developing countries such as India.

CCS Concepts: • Information systems $\rightarrow$ Data mining; Web log analysis; • Computing methodologies $\rightarrow$ Natural language processing.

Additional Key Words and Phrases: Text Mining, Government Policies, Swachh Bharat Abhiyan, Social Media Data Analysis, Sentiment Analysis, Predictive Analysis

\section{INTRODUCTION}

Government launches social and government campaigns to promote multiple government policies, health programs and services that are related to female health, mother and child care, infrastructure building, cleanliness, and financial support [2] etc. One such area where the government puts many efforts is maintaining public health. Public health is a discipline which primarily focuses on providing better health outcomes of a population. It is believed that there are numerous social, economic, chemical, physical, political and biological factors behind the cause and dispersion of a disease. The primary mode of solving these problems is applying the preventive measures, unlike the curative measures used in public health. These measures include setting up safety standards, spreading awareness among people on the risks of drug abuse, etc., educating people about myths and superstitions and vaccinating adults and children, etc.

In October 2014, government launched a national level cleanliness campaign, named Swach Bharat Abhiyan (SBA) [35] (meaning: Indian Cleanliness Campaign) which aims to clean cities, roads, public places and transportation services in urban as well as rural areas of the country. One primary aim of this campaign was to make India free from open defecation and achieve 100 percent scientific solid waste management by October 2019. Since then,

Authors' addresses: Aarzoo Dhiman, aarzoo@cs.iitr.ac.in, Indian Institute of Technology Roorkee, Roorkee, Uttarakhand, India, 247667; Durga Toshniwal, Indian Institute of Technology Roorkee, Roorkee, Uttarakhand, India, durga.toshniwal@iitr.ac.in.

Permission to make digital or hard copies of part or all of this work for personal or classroom use is granted without fee provided that copies are not made or distributed for profit or commercial advantage and that copies bear this notice and the full citation on the first page. Copyrights for third-party components of this work must be honored. For all other uses, contact the owner/author(s).

(c) 2022 Copyright held by the owner/author(s).

2375-4699/2022/8-ART111

https://doi.org/10.1145/3490503 
government has been providing budgets to build toilets, better roads, deploy dustbins in all the public places and teach about their importance in urban and rural areas of the country. After the inception of SBA, many countries applauded this idea and have shown much interest in providing resources to pace up the process. For example, Japan International Cooperation Agency (JICA), increased its focus on funding water and sanitation facilities in India [15] in October 2016. However, there has not been any satisfactory statistical details provided, other than the number of toilets deployed, which can tell the effects of this campaign on involvement and perception of common public towards cleanliness.

Every year since the inception of SBA, the government performs a nationwide survey called Swachh Survekshan (meaning: Cleanliness Survey) program to evaluate the effectiveness of the campaign. The primary modes of evaluating the effectiveness of any public health-related prevention model are to use special study designs such as retrospective or prospective observational study designs and the interventional study designs [42]. However, these traditional methods are very time intensive and costly methods and include many constraints that need more time and energy from the designer's end. Also, these experimental and quasi-experimental designs are very difficult to implement because of the limitations such as the need for critical avoidance of selection bias, randomization to the intervention, and the recruitment of the participants. These designs are generally useful on a small scale environment where the populations are low and some of these designs also need the active involvement of the population. Hence, due to all these limitations, in this study, we propose to move the attention to a faster, easier and anonymous method of studying the human perception by using the social media data also known as the Web 2.0. Many earlier researchers have used this data source to perform many public health-related tasks and have claimed that the topics, language styles thus the sentiments and opinions extracted through social media data from a specific geographical region are the strong markers of perception of people [5].

The primary advantage of social media sources in place of traditional sources is its volume and abundance. Though these sources are information-rich, it is very complex to process and analyze this data using traditional methods. Data Mining techniques have the ability to extract the knowledge from the data thereby generating better decisions [21]. There have been many research works where data from online sources such as Twitter, Google, and Facebook, etc. have been used to track the outbreak of many diseases [9], a trend in emerging topics [11] and to track the impact of a vaccine introduced in public [36] etc. The data from such online sources have made the work easier and faster without the requirement of door-to-door data collections.

Traditionally, the government of India evaluates the performance of any campaign by performing surveys in two manners. One, where the perception of the country's citizens is captured through the mode of personal interviews. Second, where the on-field development surveys are performed to evaluate the execution of documentation-based tasks. In this research work, we focus on covering both manners using social media data, i.e., Twitter. The citizens' perception has been analyzed with the help of tweets' sentiments related to a given question or topic. However, this rationale's primary bottleneck is the extraction of tweets related to a topic from a given corpus. To overcome this limitation, we propose a tweet pooling method with the help of similar words generated using word-embeddings. The whole framework takes a set of questions/topics and the Twitter corpus as input and outputs the public's general perception towards the topic. Furthermore, to fine-grain the results, the tweets' subjectivity scores have also been utilized to generate the sentiments. The proposed framework (Section 3.3) has been validated on a set of questions retrieved from public surveys [3].

In the second mode, this research work explores the impact of the city's demographic features in maintaining the standards of cleanliness set by SBA. We propose to train a regression model to predict the performance of a city in Swachh Survekshan by using the Twitter generated and the demographic features such as Number of tweets from Public Handles, Number of tweets from Government Handles, Population, Area in Square Kilometers, Female Population and Percentage decade growth in the population of the city. This will help to evaluate the place-wise (city-wise) effectiveness/performance of the campaign. The city-level demographic features have been pulled from the Indian census data (2011) [10]. However, only approx $1 \%$ of Twitter data contains geo-location information. 
To overcome this challenge, we propose to extract the city-names and location names from the tweet text. We propose to use partial keyword matching with NER tagging to extract most of the locations from the tweet text. Our study shows that most of the extracted location-names were geocoded successfully by the Google geocoding $\mathrm{API}^{1}$. We employ some simple and popular regression models to assess the city-wise survey performance using Twitter and demographic attributes. Our study found that Random Forest regression gave the best $R^{2}$ performance out of all the models. Overall, the proposed research work has proved that Twitter data can be a good indicator of government-provided information. This analysis can also help find out interesting information regarding some cities.

There is very less research work that focuses on using the data from developing countries such as India due to the limited number of users. However, India has third largest Twitter user-base ${ }^{2}$. Thus, this research work proposes that Twitter data belonging to an Indian campaign is well suited for the purpose of monitoring the impact of such campaigns over the developing country. The overall contribution of this paper can be described as follows:

- A social media data based framework has been proposed as a potential substitute for government statistical and survey data processing. This research work has processed around 9,00,000 tweets, a bigger sample size than that taken for government surveys (Section 3.1).

- A novel word embedding-based tweet pooling and subjectivity score based sentiment analysis framework has been proposed in Section 3.3 which evaluates the performance of the campaign as the citizens' point of view. This will help to reproduce the results of personal-interview based government surveys.

- A novel demographic features based prediction model has been introduced in Section 3.4 to predict the scores of the cities in India on the basis of their performance in maintaining cleanliness. This helps to reproduce the results of government documentation based surveys.

- The Twitter and demographic data of cities have been analyzed to compare and contrast the performances of different cities in the country in terms of maintaining cleanliness. This analysis helps to identify some interesting patterns among the cities (Section 4.2.1).

The rest of the paper is organized as follows. Section 2 contains related work, which discusses some of the previous work done in this field. Section 3 contains the proposed methodology, which discusses the details on data collection, pre-processing and its analysis. Section 3.3 presents the steps involved in developing the Twitter text data based citizens' feedback evaluation module of the framework. Section 3.4 presents a demographics feature based prediction model that can evaluate the performance of cities in maintaining cleanliness. Section 4 provides the details on the ground truth data collection and the experimentation performed. Section 4.2.1 also provides some details on cluster analysis of the cities to generate the patterns and trends followed by cities' demographic features in terms of maintaining cleanliness. Finally, the paper ends with the conclusion and references.

\section{LITERATURE REVIEW}

There has been much research work where social media data has proven to be a great alternative to the data collected manually or through records. These data sources are preferred over the traditional data sources for solving multiple data-centric problems due to the advantages such as faster and easier accessibility with minimal efforts and huge volume and variety of data. The primary contributions of social media in the field of public health are tracking the outbreak of a disease [4] which includes modeling and predicting the spread of disease in a population, tracking the trend in emerging topics related to different health issues [11], predicting the presence of depression or mental health problems in the social media users [12], information sharing patterns of alcohol consumption among users [24] and monitoring the effectiveness of many interventions introduced

\footnotetext{
${ }^{1}$ https://developers.google.com/maps/documentation/geocoding/overview

${ }^{2}$ https://www.statista.com/statistics/242606/number-of-active-twitter-users-in-selected-countries
} 
among population to improve public health situations which include monitoring the impact of vaccinations and drugs introduced in the population [36].

\subsection{Social Media and Public Health}

The most notable contribution in predicting the spread of disease using web data was made by Google in 2009 . Here, Ginsberg et al. introduced Google Flu Trends (GFT) [18], a web service that helps in early detection of the spread of a disease in a given population. They monitored the daily queries related to Influenza-like Illness (ILI) posted by users on online search engine i.e. Google. Similarly, many other online data sources such as Twitter [9], Yahoo! [30], medical websites [20] and their combinations [27] have been explored through the years to perform surveillance which use different techniques such as Least Absolute Shrinkage and Selection Operator (LASSO) [22], BOLASSO [23] and classification tools like Support Vector Machine (SVM) [4] etc.

There may be many socio-economic, environmental, chemical and biological factors behind the causes and spread of disease. For example, poor waste management, sanitation, and sewage conditions, etc. lead to many foodborne, air-borne and water-borne diseases. Thus, the government introduces preventive measures to eliminate these factors and improve public health. The effectiveness of these preventive measures also needs to be tracked so that appropriate actions could be taken. In 2010, Scanfeld et al.[36] examined the data from Twitter to track the misuse and misunderstanding related to the use of antibiotics in society by using content analysis techniques. Later in 2017, Shah et al.[38] traced the change in behavior of users search data before and after the introduction of Rotavirus and Norovirus vaccination in the US, UK, and Mexico by using the data from Google quantified Internet Query Share (IQS).

\subsection{Social Media and Governance}

Global Digital Reports 2019 [1] have shown that the number of online users is increasing with an average of more than one million new users per day i.e. 11 users per second. With mobile accounting for increasing share of online activities, the users are spending almost half of their online time on mobile phones. According to this study, though there is an increase of $8 \%$ in the number of social media users worldwide there is a $10 \%$ increase in the number of mobile social media users worldwide since last year. Social media has made it easier for the common public to raise their voices and express their views on the multiple services provided by social and government organizations [7].

The decision making in governance has also benefitted very much with the increased use of social media by citizens [43]. The primary benefits achieved in e-governance are less cost, high information penetration among citizens e.g. high crisis communication, humanizing the government for public and thereby increasing the mutual trust. This also helps in increasing transparency and citizen participation. In [16], authors have shown that the mayors can act as the bridge in the Twitter network of public services with the help of a case study of Seoul, Korea. This bridge then helps in increasing the responsiveness towards governance. In [13], authors developed a typology for government communications and concluded that it belongs to the sphere of symbolic and presentational exchanges.

There are many different applications of social media data analysis in governance as per the literature. In [31], the authors analyzed the data related to 18 social events available from Flick. They integrated a supervised topic model in a boosting framework. In [26], authors provided a framework to analyze the citizens' opinion regarding municipal administrators using an online platform of a city in China. In [34], authors identify the topics raised by citizens related to solid waste management on Facebook. They used Latent Dirichlet Allocation (LDA) for identifying the keywords such as recycling, containers, and inquiries from the data corpus.

In this research work, we focus on monitoring the influence of Swachh Bharat Abhiyan (SBA) on people using Twitter data. SBA was first launched in 2014 and since then very less research work has been done related to 
it. In 2015, Sahil Raj et al. [33] collected tweets related to SBA and performed simple sentiment analysis to find out the perception of Indian citizens towards SBA. Later in 2016, Devendra et al. [41] tested their sentiment analysis tool Senti-Meter on Twitter data related to SBA. They studied 1200 tweets collected for the period of January 2016 to March 2016 and performed manual tagging to evaluate the accuracy of their tool. Both of these research worked on very less number of tweets and did not consider any other demographic details of the Indian cities and states. The existing research works have used only sentiment analysis as a tool to measure to public perception. These models have not tried to generalize the idea of computing the public perception to assess the overall situation of the campaign in the country. Evaluating the effectiveness of a government campaign comes under 'Intervention Assessment' i.e. monitoring the impacts of any intervention introduced in the population. There has not been much research work related to using social media data such as Twitter to track the impact of a government campaign. In this research work, Twitter data has been processed in three dimensions to capture the overall impact of this campaign. The dimensions captured for our work include $(i)$ studying the perception of people, (ii) evaluating the overall performance of different cities in the country and (iii) analyzing the Twitter and demographic information related to the cities to find out some interesting patterns among these cities.

Inspired by the previous discussion, it becomes a challenge to process all this data to generate important knowledge. This challenge introduces the need for frameworks and models which are capable of extracting such knowledge and transferring it to the policymakers and organizations. In this work, we propose natural language processing (NLP) based models to generate quality information from the data and providing a structure to the opinions of public/citizens. The proposed citizen feedback predictor given in Section 3.3 will help to provide structure to the public opinions towards SBA. Similarly, the demographic features based performance predictor given in Section 3.4 will help in extracting the information from raw facts and figures. The NLP based models help us to achieve these goals.

\section{PROPOSED METHODOLOGY}

In this section, a brief description of the steps involved in the proposed framework has been given. The framework comprises of three primary modules i.e. (i) Dataset collection and pre-processing (ii) Citizen's feedback evaluation, and (iii) Demographic feature based performance evaluation model. Section 3.1 provides a detailed description of the data collection and pre-processing procedure. Section 3.3 presents the steps involved in developing the Twitter text data based citizens' feedback evaluation module of the framework. Finally, Section 3.4 provides the framework for demographic features based city-wise performance prediction model.

\subsection{Twitter Dataset Description}

With the growth of the internet more and more personal data is available publicly. It has been seen that with the increase in the usage of social media, people are more aware of the problems of society through some social groups, activities, and events that are occurring on a daily basis. These sources help the users to provide their point of views related to many events and thus public need not rely on the news networks, governmental speeches or some hearsay.

In this study, Twitter data has been used to deliver insights on the performance of different cities in terms of public perspective and on-field development. The steps involved in data collection and pre-processing for both the modules of the proposed framework have been given in this section. The Twitter Streaming API was used to collect the real-time Twitter data all-over the world. The Twitter API was able to collect around 9,00,000 tweets for the period of December 1, 2017 to August 31, 2018 using a set of 82 keywords pertaining to different topics related to SBA such as "SwachhBharatAbhiyan", "MyClean-India", "MyCityMyPride" and "StopOpenDefecation" which further belong to different topics related to SBA such as "rural area related", "defecation-related" and "cities related" etc. The types of keywords used and sample description of tweets have been given in Table 1. 
Table 1. A brief description of the Twitter data collected for the period of December 2017 to August 2018

\begin{tabular}{|c|c|c|c|}
\hline Description & $\begin{array}{l}\text { Hashtags } \\
\text { Examples }\end{array}$ & $\begin{array}{l}\text { Tweets } \\
\text { Example }\end{array}$ & $\begin{array}{l}\text { Number } \\
\text { of Tweets }\end{array}$ \\
\hline General & $\begin{array}{l}\text { \#Swachh Bharat Abiyan } \\
\text { \#MyCleanIndia } \\
\text { \#SwachhSurvekshan }\end{array}$ & $\begin{array}{l}\text { @marineravin:@tavleen } \\
\text { singh anything you wanna more to add abt swachh } \\
\text { Bharat Abhiyan... @sanjayuvacha @amitmehra }\end{array}$ & 644,574 \\
\hline Toilet Related & $\begin{array}{l}\text { \#OpenDefecation } \\
\text { \#MyCityMyPride } \\
\text { \#StopOD }\end{array}$ & $\begin{array}{l}\text { @paramiyer } \\
\text { : Congratulations to Team @swachhbharat. } \\
\text { Tirunelveli district in Tamil Nadu has been declared } \\
\text { \#OpenDefecationFree. } \\
\text { @lezlietripathy: }\end{array}$ & 53,692 \\
\hline Cities Related & $\begin{array}{c}\text { \#SwachhUP } \\
\text { \#SwachhJhar } \\
\text { \#CleanDelhi } \\
\text { \#SwachhVersova }\end{array}$ & $\begin{array}{l}\text { Participated in Cleaning \#Vesave } \\
\text { Beach Today. An initiative by @AfrozShah1 Support } \\
\text { @Dev Fadnavis @AUThackeray } \\
\text { Today. \#SwachhBharat \#Swachhmaharashtra } \\
\text { \#swachhversova }\end{array}$ & 49,472 \\
\hline Rural Area Related & $\begin{array}{c}\text { \#ZSBP } \\
\text { \#SbmZSBP } \\
\text { \#SwachhBharat- } \\
\text {-MissionGrameen }\end{array}$ & $\begin{array}{l}\text { @kishanganjzsbp: } \\
\text { Morning follow up and pit digging in Gachpada } \\
\text { Panchayat \#ZSBP \#SwachhBharat } \\
\text { \#SwachhBihar \#SBMGramin } \\
\text { @SwachhBihar @LSBA Bihar @swachhbharat }\end{array}$ & 173,736 \\
\hline
\end{tabular}

3.1.1 Data Pre-processing. The tweets collected contains a lot of information. This whole information may or may not be relevant for the study. The improved quality of raw data helps in providing considerably high-quality results. Thus, the Twitter data set has been first pre-processed. The steps involved have been given further in the section.

First the tweet text, user ID and the date of tweet creation are segregated from the tweets. Then the tweet text is cleaned by removing the stop-words such as is, the, on, and at etc. from the tweet. We use the standard list of stop-words available with NLTK python library to search for stop-words from the text-sentences. The tweet text itself is subjected to other information due to the use of informal and idiomatic language by the users e.g., emoticons, hyperlinks, images, and videos, etc. We remove such tokens from the tweets with the help of regular expressions. Then, to improve the quality of the results stemming has been performed on tweet text. The stemming helps in bringing the tokens to their root forms. The resultant cleaned tweets are then processed to obtain the information related to cities and states.

\subsection{Location Extraction from Tweets}

The Twitter corpus is further processed to assess the involvement of people in different cities and states in India. The formal method of getting information about a place through the tweets is either through the geo-location or profile information specified in a tweet. In the case of geo-locations, only around $1 \%$ of the tweets contain geo-locations and this data cannot be considered enough to provide accurate results. In the case of user profile information, it cannot be confirmed that one's tweet is about the same place as given in one's profile. For example, for tweets such as "RT @Swacchbegusarai: Door to door visit and triggering to increase uses of toilets in Fatehpur Panchayat@swachhbharat @LSBA_Bihar\#ZSBP htâĂę" posted by the Twitter handle "CleanupTN", geocoding gave us the name of place "TamilNadu" whereas the tweet actually talks about district "Begusarai" and village "Fatehpur" which belongs to state "Bihar". 
To overcome the limitation of inconsistency in location found from user-profile and the textual content of tweet, we extract the location information from the tweets using Named Entity Recognition (NER) and partial keyword matching [14]. The NER module provided with the python NLTK ${ }^{3}$ library helps in facilitating the labeling of keywords into corresponding Part-of-Speech (POS) and locate and classify the named entities in a sentence. We used noun phrase chunking using regular expression $N P:\{\langle D T\rangle$ ? $\langle J J\rangle *\langle N N\rangle\}$ to identify the named entities. Furthermore, we performed IOB tagging on the chunked noun-phrases where B stands for beginning, I stands for inside and O stands for end of the sentence. Finally the python function nltk.ne_chunk() helped in labeling the tokens with corresponding named entities as PERSON, ORGANIZATION, and GPE. The GPE tag represents the geo-graphical position. Furthermore, we also explored the python Spacy ${ }^{4}$ module to generate the geo-locations from the text. Spacy is an Industrial Strength Natural Language Processing library in python. This library provides off-the-shelf functions to be applied on the text data ranging from tokenization, tagging, parsing, lemmatizing etc. The spacy module was simpler and more accurate as compared to the NLTK module. The NLTK identified approximately 1,10,554 non-unique locations, whereas spacy identified 4,90,752 non-unique locations. The named entities which are not locations are ignored by the geo-coding software, thus there is no need to identify if the named entity represents a location or not. Figure 1 provides the word-cloud visualization of the named entities tagged as GEP by NLTK and Spacy respectively. Figure 1 shows that the most commonly identified locations are Delhi, Chhattisgarh, Indore, Odisha, Jammu etc. NLTK and spacy returned different results for the same tweets, thus we take the set union of the names returned by both the modules.

However, the manual analysis of some of the sentences showed that the GPE tag failed to tag many locations via the text. For example, in tweet text "Motivate people's install swachhata app To participate to muzaffarpur smart city@swachhbharat @Swachhjhar @SwachhMansa@Muzaffarpur_SCM @swachhhyd https://t.co/p20yB7A2FZ", NLTK labeled motivate as GPE and failed to recognize muzaffarpur as place. Thus to overcome this limitation, we propose to employ partial keyword matching in the tweet text using the names of 4023 small and large cities available through the government survey and census data [10] [40]. The list may not be exhaustive, however, contained all the names for which government of India has provided Swachh Survekshan results.

To perform partial keyword matching, we calculate the partial match between the tweet text token and the city or state name and then geocode only those entities for which the partial match comes out to be greater than $95 \%$. The partial match helps to identify the location from the tokens present in concatenated form and the having some spelling errors, such as 'SwachhMansa', 'SwachhOrissa' and 'swachhOdisha'. The mathematical formulations of the steps involved are as follow. Let $S$ be the set of name of states and union territories in India. So, $S=\left\{S_{1}, S_{2}, \ldots S_{n}\right\}$, where $n$ is the number of states and union-territories. Let $C$ be the set of name of cities and towns in India. So, $C=\left\{C_{1}, C_{2}, \ldots C_{m}\right\}$, where $m$ is the number of cities and towns in India. We used around 4023 of the city names for our analysis. A dictionary $C S$ consists of the names of cities and their corresponding state name. So, $C S=\left\{C_{i}: S_{j}\right\}, 1 \leq i \leq n, 1 \leq j \leq m$ such that $C S\left[C_{i}\right]=\left[S_{i}\right]$.

Let $T_{i}$ be the twitter corpus and frequency score $F\left(C_{i}\right)$ of a city $C_{i}$ is calculated by using the formula given in Equation (1). A tweet $t_{j}$ in the corpus is given a score 1 if name of city $C_{i}$ is present in that tweet and 0 otherwise as given in Equation (2). We took care of the partial matching of names in the tweets as well as given in Equation (3).

$$
\begin{gathered}
F\left(C_{i}\right)=\Sigma \Sigma F\left(C_{i}, t_{j}\right), \forall i \forall j \\
\text { where, } F\left(C_{i}, t_{j}\right)=1 \quad \text { if } C_{i} \in t_{j} \text {, Otherwise } 0 \\
\text { PartialMatch }=\left(\frac{2 M * 100}{T}\right)
\end{gathered}
$$

\footnotetext{
${ }^{3}$ https://www.nltk.org

${ }^{4}$ https://spacy.io
} 


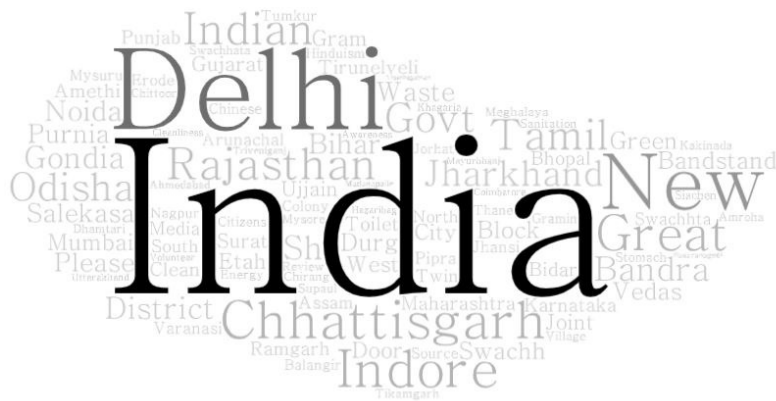

(a) NLTK

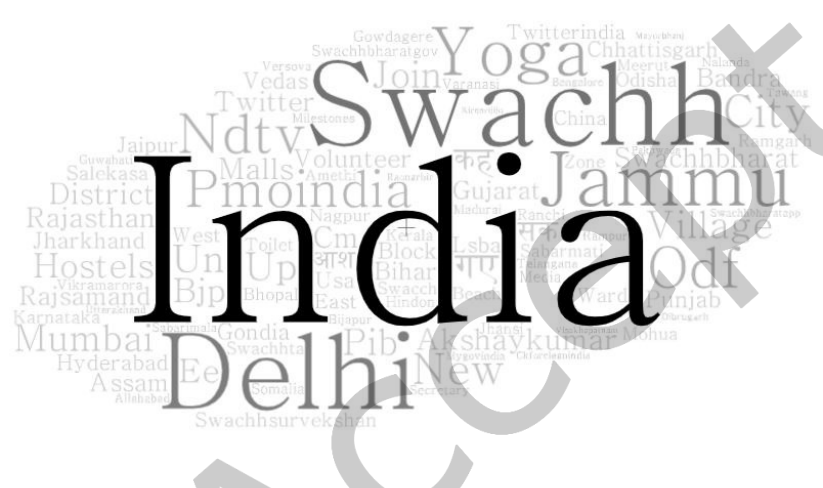

(b) spacy

Fig. 1. The word-cloud visualization of the named entities tagged as location by off-the-shelf modules NLTK and spacy

where, $M$ is the number of matches and $T$ is the total number of elements in the string. Similarly, the frequency score of a state is calculated by using the frequency score of the state and its corresponding cities. More formally, frequency score of a state $S_{j}$ is equal to the sum of frequency score of the state $S_{j}$ and sum of frequency scores of all the cities $C_{i}$ for which $C S\left[C_{i}\right]=S_{j} \forall i$ as given in Equation (4) and Equation (5).

$$
\begin{gathered}
F\left(S_{i}\right)=\Sigma \Sigma F\left(S_{i}, t_{j}\right)+\Sigma F\left(C_{k}\right), \forall i \forall j \forall k \text { and } S C\left[C_{k}\right]=S_{i} \\
\text { where, } \mathrm{F}\left(S_{i}, t_{j}\right)=1 \quad \text { if } S_{i} \in t_{j}, \text { Otherwise } 0
\end{gathered}
$$

By using this technique, we were able to collect results even for very small cities and towns which were not mentioned in the userâÁźs profile or geo-location. The number of tweets collected per city and state by using geoparsing was also found to be higher than by using geocoding the tweets.

A city may consist of some people who are highly active on twitter and some who hardly use it. Twitter corpus when analysed using the absolute number of tweets only give cumulative results for a particular place. Also, more the population of place, more is the number of tweets collected. However, when the number of tweets is normalized by the factor of population of the place, this gives us the intensity of tweets per person in a given city $C_{i}$. We normalize the score value of a place by dividing it with the population of the place (i.e. per capita score) 


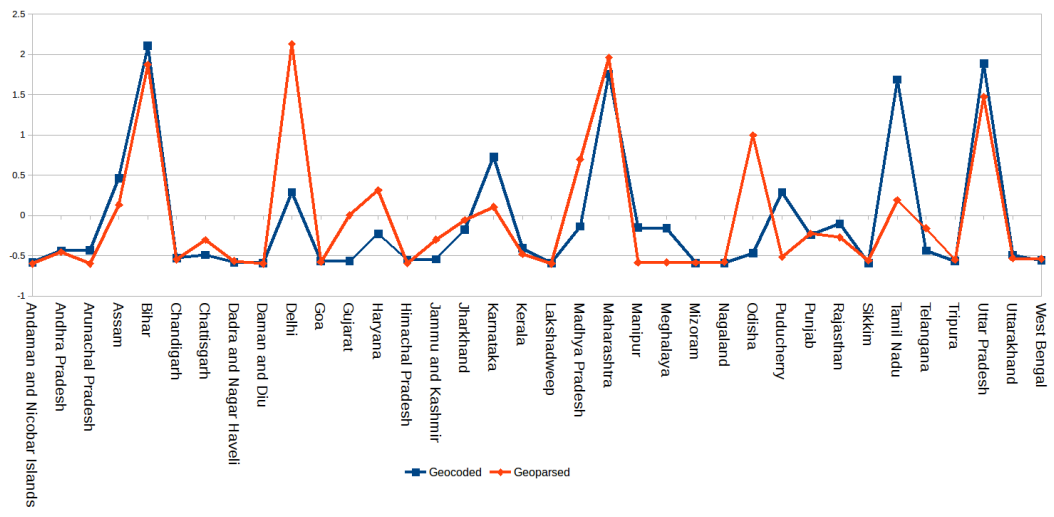

Fig. 2. Normalized number of tweets collected by using geocoding and geoparsing for all states and Union Territories in India

as given in (6).

$$
N\left(C_{i}\right)=\frac{F\left(C_{i}\right)}{P\left(C_{i}\right)}
$$

where, $P\left(C_{i}\right)$ is the population of city $C_{i}$. To provide a pictorial depiction, we normalized the number of tweets collected by geocoding and geoparsing by using $\mathrm{z}$-score normalization. Figure 2 gives a line plot for the number of tweets for different states on the sample data for the time period of March 1 to March 15, 2018. Figure 3 provides the state-wise heat-map plot to represent the density of tweets, where red stands for maximum number of tweets and green stands for lowest number of tweets.

SBA is a national level campaign and the government is making many efforts to promote it. There are dedicated twitter accounts which promote SBA in their local communities e.g., SwachhUP, Swachh_fhar and MoHUA_India etc. However, because of their bot-like behaviour, these dedicated handles cannot be considered relevant to track the public perception. So, the tweet texts have been categorized into government generated tweets, which includes the tweets posted by using these dedicated handles and public generated tweets, which includes all the rest of the tweets. These dedicated handles were spotted from the corpus using the keyword search [14].

\subsection{Predicting Citizen Feedback}

The traditional method of assessing the effectiveness of a campaign includes the retrospective surveys which are based on the personal interviews. These interviews help to gather citizens' opinions and is considered one of the important types of surveys performed by the government. These surveys are vital in the view that these evaluate the likes and dislikes of the citizens of a country towards any campaign/event. However, the establishment of the survey questionnaire, interviewers, interview infrastructure and the consent of interviewees incurs high cost, time and manpower. Automatizing the process of gathering the people's perception through the social media can help eradicate the limitations of the traditional survey methods. In this section, a social-media based framework has been proposed to predict the citizen feedback while considering the Swachh Bharat Abhiyan (SBA) as the case-study.

3.3.1 Word Embedding based Tweet Pooling. In this section, the framework to evaluate the perception of people towards a campaign using the Twitter data has been provided as illustrated in Figure 4. In the traditional methods, retrospective surveys prepare a questionnaire to be answered by common public. A sample question in context of SBA can be "What is the perspective of citizens towards the construction of toilets in a rural area?" Here the 


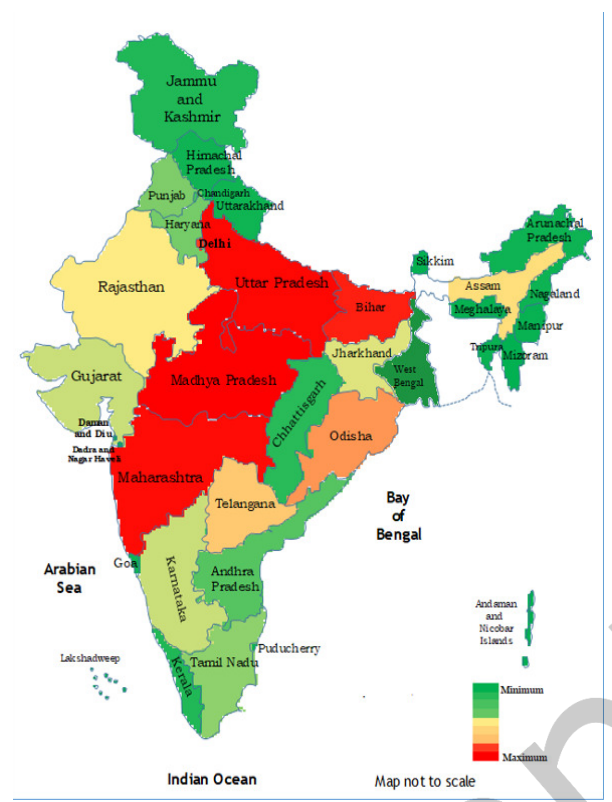

Fig. 3. Heatmap of India generated by processing tweets to study their demographic distribution.

primary topic of concern is toilet-construction and rural-area. Similarly, every question can be represented as a set of topics, consisting of topical-keywords. Similarly, every tweet can be represented as the set of tokens $t=\left\{t_{1}, t_{2}, \ldots, t_{i}\right\}$, where $t_{i}$ is the $i^{t h}$ token generated after pre-processing the tweet $t$. The proposed framework is based on the rationale that these question-topics can be used to extract the tweets mentioning the topic

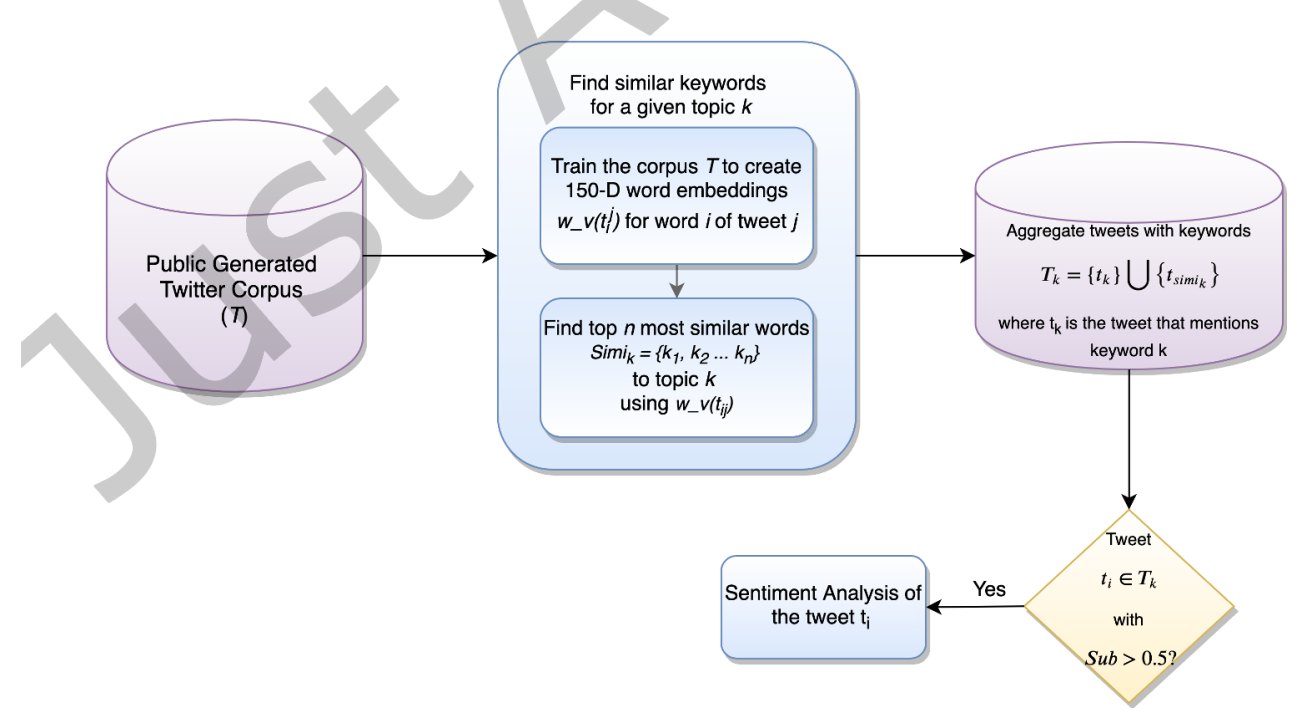

Fig. 4. Block diagram giving the steps involved in the approach to reproduce the result of personal interview based surveys 
automatically. This will help to reduce the cost and time of asking the question from common public and then waiting for the responses. The cumulative sentiment of the set of tweets extracted for a question/topic can be further utilized to generalize the response of public towards the question. The steps involved in the proposed method have been elaborated further in the section.

First, the Twitter corpus is divided into government generated tweets and the public generated tweets as explained in Section 3.1.2. Next, a tweet pooling (Definition 1) based approach has been used to cluster the tweets belonging to a given question-topic $k$. For example, the topical-keywords in $k$ for a general question "What is the perspective of citizens towards the construction of toilets in a rural area?" will be $k=\left\{\right.$ toilet $^{\prime}$, ' $^{\prime}$ onstruction',' rural $\left.^{\prime}\right\}$.

Next, we use Continuous Bag of Words (CBOW) model of Word2Vec [28] to generate word vectors for our corpus in an unsupervised manner. These word vectors will be used to calculate the semantic-similarity between the topical-keywords and the tweet-tokens. We used CBOW model of Word2Vec because this is a simple language representation model having better performance for the word semantic similarity extraction tasks [29]. The semantic similarity between any two tokens has been calculated using the cosine similarity between any two word vectors (generated using $\mathrm{CBOW}$ ) as given in Equation 7. The CBOW model has been trained on our Twitter corpus. The training model generates a 150 dimensional word vector representation $w_{-} v\left(t_{i}^{j}\right)$ for token $i$ of tweet $j$. Let a topic (survey question) $K$ can be represented as set of $n$ topical-keywords i.e. $k=\left\{k_{1}, k_{2}, \ldots, k_{i}\right\}$, where $k_{i}$ is the $i^{t h}$ keyword that defines topic $k \in K, K$ being the set of all the questions/topics. Similarly, the tweet can be represented as set of $m$ tokens i.e. $t=\left\{t_{1}, t_{2}, \ldots, t_{j}\right\}$, where $t_{j}$ is the $j^{t h}$ token that defines tweet $t \in T$, $T$ representing the set of tweets in the corpus. The similarity between the topical-keywords and tweet-tokens i.e. $\operatorname{sim}(k, t)$ has been calculated using Equation 8 . This helps to identify the tweets sharing similar context as the given topical-keywords. Let $p_{k}$ is the set of pooled tweets which belong to the same topic as $k$ such that $T_{k} \subseteq T$. A tweet $t$ is added to $T_{k}$ if $\operatorname{sim}(k, t)$ is higher than other $\operatorname{sim}(K-\{k\}, T-\{t\})$. The set of tweets $T_{k}$ with highest scores in $\operatorname{sim}(k, t)$ are then considered to be belonging to the same topic $k$. The set $T_{k}$ is the pooled tweets representing the topic $k$ and these are further used to evaluate the perception of the citizens towards the topic $k$. The detailed steps have been given in Algorithm 1.

$$
\begin{aligned}
& \operatorname{cos\_ sim}(u, v)=\left(1-\arccos \left(\frac{u \cdot v}{\|u\|\|v\|}\right) / \pi\right) \\
& \operatorname{sim} \_\operatorname{score}(k, t)=\frac{1}{m} \frac{1}{n} \sum_{i} \sum_{j} \cos \_\operatorname{sim}\left(k_{i}, t_{j}\right)
\end{aligned}
$$

Continuous Bag of Words. The proposed module uses the Continuous Bag of Words (CBOW) [28] model of Word2Vec to find out the similar words to a given topic $k$. The CBOW model predicts the target word corresponding to a given window of context words. This model is used because it shows a better accuracy over the Skip-gram model of Word2Vec in case of frequent words. Equation 9 provides the mathematical formulation for calculating the output on the output layer. Figure 5 provides the pictorial representation of the same. Here, the input layer consists of the tweet tokens/words one-hot encoding i.e. $\left(w_{1}, w_{2}, \ldots, w_{C}\right)$, for window size $C$ and vocabulary size $V . W$ and $W^{\prime}$ are the weight matrices between input and a hidden layer of dimension $V \times N$, and hidden and an output layer of dimension $N \mathrm{x} V$ respectively. Further, the back-propagation model is used to update the weight matrices of the model using the loss function as given in Equation 12.

where,

$$
y_{j}=p\left(w_{y_{j}} \mid w_{1}, \ldots, w_{c}\right)=\frac{e^{u_{j}}}{\sum_{j^{\prime}=1}^{V} e^{u_{j}^{\prime}}}
$$

$$
u_{j}=v_{w_{j}^{T}}^{\prime} \cdot h
$$




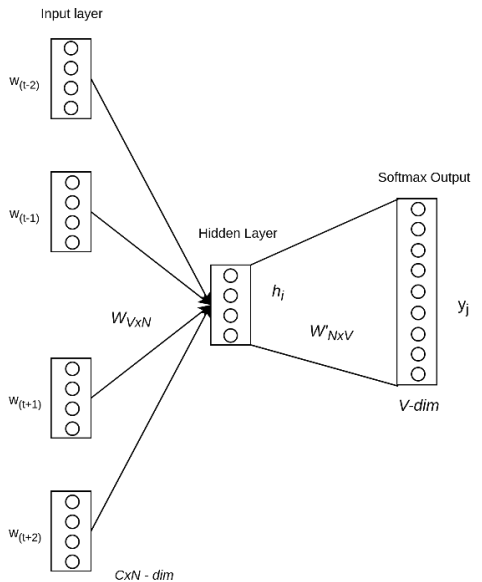

Fig. 5. The deep network consisting of input layer, hidden layer and the output layer used in the CBOW model of Word2Vec

where, $v_{w_{j}^{T}}^{\prime}$ is the $j_{t h}$ column of output matrix $W^{\prime}$

and,

$$
\begin{gathered}
h=\frac{1}{C} W\left(\sum_{i=1}^{C} x_{i}\right) \\
E=-\log p\left(w_{O} \mid w_{I}\right)
\end{gathered}
$$

where, $w_{O}$ and $w_{I}$ are the output and input words/tokens respectively.

Figure 6 gives the 2-dimensional t-SNE plot [44] representation of the word vector learned through the tweets related to SBA. The figure shows the six topics related to SBA, chosen randomly as examples: i.e. 'Toilet', 'Defecation', 'Municipality', 'Dengue', 'Disease,' 'Garbage'. Generally, all the six keywords are causally related to each other, such as toilets are constructed to eradicate open defecation, and municipality is responsible for the garbage collection etc. This type of similarity is also visible in the Figure 6. It is visible from the given figure that keywords related to toilet and defecation are very close, similarly dengue and diseases are very close on the plot. Words related to municipality are found equidistant to that of toilet \& defecation and diseases \& dengue. Finally, the keywords related to garbage are found near to municipality and diseases. This shows that the Word2Vec model captures the relationships very well here.

3.3.2 Subjectivity Score based Sentiment Analysis. The resultant tweets corresponding to each topic are analyzed to find out the sentiments of each tweet using lexicon based sentiment analysis tool (Defintion 2) in python 2.7. Here, the polarized lexicon corpus of the words such as Word Sense Disambiguation, Senti Word Net [6] and word occurrence statistics using movie review corpus has been used to assign polarity score of each token in the tweet. The overall average of the polarity score of the tweet has been used to evaluate the opinion of the tweet. Each tweet has been classified into three opinions: positive, negative and neutral. However, the performance of this sentiment analysis tool is limited by the type and quality of input data/tweets and the lexicons provided. Thus, information which indicates the better emotional content in a text can improve the quality of the output. Thus, the subjectivity score ${ }^{5}$ based sentiment analysis of the tweets have been performed.

\footnotetext{
${ }^{5}$ https://planspace.org/20150607-textblob_sentiment/
} 


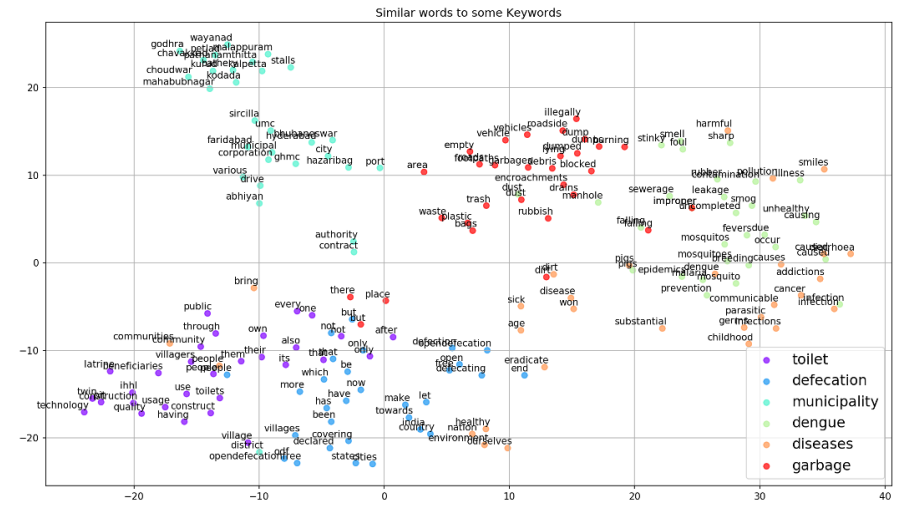

Fig. 6. A 2-D plot representation of the similar words generated for a set of keywords using Word2vec

Definition 2. Sentiment Analysis: Sentiment analysis is the task to detect the opinions and sentiments from different topics from the given textual data and classifying the inclination of opinions towards a particular emotion. The basic sentiment analysis classifies text into positive and negative sentiments.

Definition 3. Subjectivity Analysis: Subjectivity analysis is the task to detect the private states of the topic in the text. These private states can be emotions, sentiments, beliefs and opinions etc.

The Subjectivity here means the power of a text to show any particular sentiment or in other words, sentences which express some personal feeling, opinion, belief or desire, etc. are called subjective. It is known that the presence of adjectives and adverbs is a good indicator of text subjectivity. This includes the keywords such as "poor", "interesting" and "awesome" etc. So, subjectivity analysis (Definition 3) has been performed by calculating the subjectivity score of the tweets by using polarity scores of the adjectives and adverbs found using the Part of Speech (POS) tagging of the tweets. POS refers to marking the tokens in the text/tweets as corresponding to a particular part of speech e.g., noun, adjective, and verb etc. The polarity scores have been taken from the Word Sense Disambiguation, Senti Word Net [6] and word occurrence statistics using movie review corpus corpora. Subjectivity scores give the quantitative measure of the subjectivity of a sentence. Subjectivity scores $(S u b)$ ranges from 0 to 1 where $S u b=0$ means sentence is $100 \%$ objective and $S u b=1$ means sentence is $100 \%$ subjective.

The topic pooled corpus $T_{k}$ is further filtered using the subjectivity scores of the tweets in $T_{k}$ as shown in Algorithm 1 (Line 19-26). A user defined threshold on subjectivity score (sub_threshold) has been used to remove the tweets from $T_{k}$ if the subjectivity value of $t$ is less than the given threshold. Finally, the sentiments of the tweets in topic pooled corpus $T_{k}$ are used to calculate the sentiment of topic $K$. In this work, we use two thresholds i.e. $s u b \_t h r e s h o l d=0$, and sub_threshold $=0.5$. The threshold value of 0.5 will tend to return higher subjective tweets as compared to the threshold value of 0.0 . However, 0.5 represents an average value of subjectivity, thus to further generalize the analysis, we evaluated the results with sub_threshold $=0.5$ included and excluded. This gives rise to two more thresholds i.e. sub_threshold $=0.5$ (where tweets with exactly 0.5 score are included) and $s u b$ threshold $>0.5$ (where tweets with exactly 0.5 score are removed). The final allowed values of sub are: $s u b=0, s u b>=0.5$ and $s u b>0.5$. These values have been considered to calculate the sentiment scores. The accuracy of the sentiment analysis has been calculated using the manually annotated Twitter corpus of approximately 4000 tweets. The F1-score of the sentiment analysis was found to be $70.2 \%$. The validation of the proposed framework has been done using the ground truth data as explained in Section 4.1 and 4.2. 


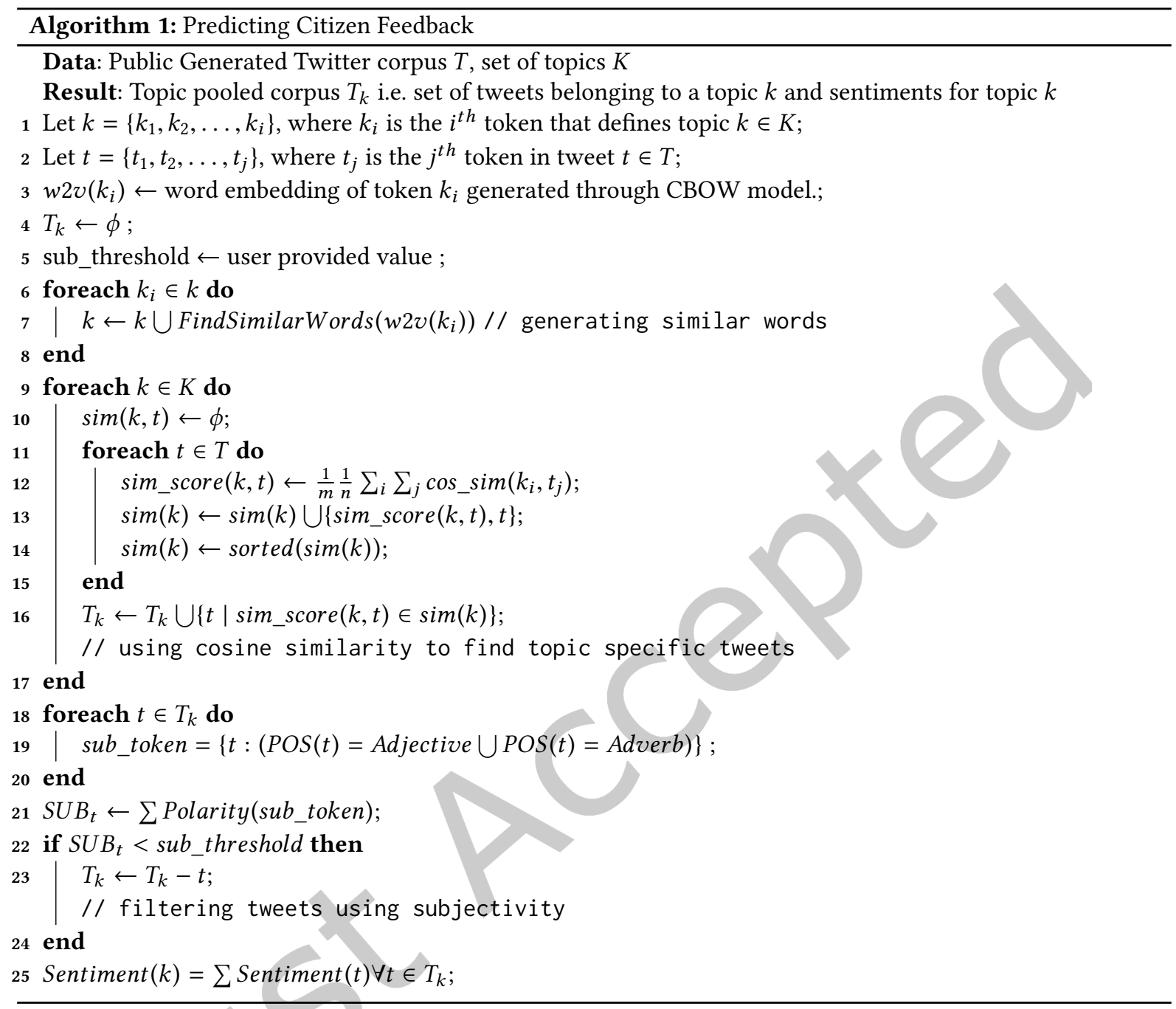

\subsection{Demographic Features based Performance Predictor}

Traditionally, the assessment of any government campaign is done through the citizens' feedback and on-ground inspections. In this section, we utilize the social media data and the demographic features to evaluate the effectiveness of a campaign using regression. The proposed model framework has been provided in Figure 7. The proposed approach addresses limitations of traditional methods which require time, cost and labor for data collection and processing.

3.4.1 City-wise Performance Data. The national level survey for SBA called Swachh Survekshan is done every year since the inception of SBA to evaluate the performance of different areas in the country in maintaining cleanliness. In 2019, a total of over 2700 assessors from Karvy went on the ground to visit over 4203 Urban Level Blocks (ULBs), spending over 0.23 million person-days from $4^{\text {th }}$ January to $10^{\text {th }}$ March 2018. As a result of the 


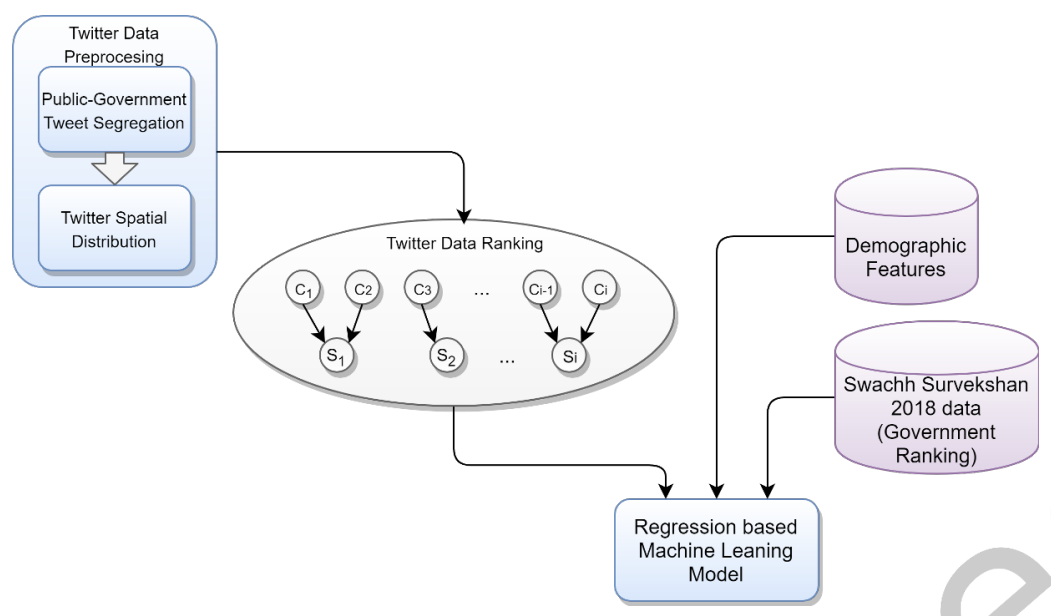

Fig. 7. Block diagram giving the steps involved in the approach to predict the performance of the cities in maintaining cleanliness

survey, all the cities were given ranks on the basis of total scores i.e. Overall Marks (OM) which is the sum of scores for the following parameters:

(1) Service Level Progress (SLP): SLP involved collection of documentary evidence provided by ULBs on the activities they undertake under SBA.

(2) Direct Observation (DO): In case of DO status of cleanliness was verified through on-ground observation at sample locations.

(3) Citizen Feedback and Swachhata App (CFSA): In case of CF direct feed-back from the citizens of each ULB was collected through face-to-face facts, outbound calling, and an online survey. And for SA, marking was based on the number of downloads and complaint redressal.

Government Ranks. For this study, Swachh Survekshan 2018 (SS2018) has been used as the ground truth for scoring the cities on the level of cleanliness [40]. For this purpose, the cities have been given the ranks for each type of scores, where a high score means a lower rank. The rankings have thus been called SLP-Rank, DO-Rank and OM-Rank respectively. Here, the CFSA score can be ignored as this has already been considered in the previous section.

The government scoring mechanism uses three types of scores to generate a performance evaluation of a city. However, all three scores were not available for all the 4023 cities online. Thus, Overall Scores (OM) of all the cities have been used as the target attribute for the supervised training of the respective prediction model.

Twitter Ranks. The cities are also ranked on the basis of the number of tweets generated from a location given in 4023 cities used for the survey. The process of extracted tweets from a given location has been described in Spatial Distribution in Section 3.1.2. To maintain symmetry among the Twitter data and the government data, the cities have been ranked using three types of scores generated using the Twitter data. These ranks have been generated by scoring the cities based on the number of tweets generated through government handles and the public handles. Below given is the brief description of all these ranking schemes used.

(1) Rank-Abs: Ranks given to the cities on the basis of the total absolute number of tweets.

(2) Rank-Abs-G: Ranks given to the cities on the basis of the absolute number of tweets generated by using the government handles. 
Table 2. The correlation among the government given ranks and Twitter data generated ranks

\begin{tabular}{lccc}
\hline $\begin{array}{l}\text { Govt } \\
\text { Ranks/Twt }\end{array}$ & Rank-Abs-U & Rank-Abs-G & Rank-Abs \\
Ranks & & & \\
\hline SLP & 0.654 & 0.171 & 0.592 \\
DO & 0.502 & 0.056 & 0.406 \\
OM & 0.54 & 0.056 & 0.461 \\
\hline
\end{tabular}

(3) Rank-Abs-U: Ranks given to the cities on the basis of the absolute number of tweets generated by using the public handles.

3.4.2 Correlation Analysis. After generating the Twitter ranks and the Government ranks for the given 4023 cities, a correlation analysis between them has been performed to further find out the similarity among the ranks. Table 2 gives the Spearman's rank correlation coefficients as given in Equation (13) among all the ranks.

$$
r_{s}=\rho_{r g_{X}, r g_{Y}}=\frac{\operatorname{cov}\left(r g_{X}, r g_{Y}\right)}{\sigma_{r g_{X}} \sigma_{r g_{Y}}}
$$

where, $X_{i}$ and $Y_{i}$ are converted to rank variables $r g_{X}$ and $r g_{Y} . \rho$ denotes the usual Pearson correlation coefficient [37], but applied to the rank variables. $\operatorname{cov}\left(r g_{X}, r g_{Y}\right)$ is the covariance of the rank variables. $\sigma_{r g_{X}}$ and $\sigma_{r g_{Y}}$ are the standard deviations of the rank variables.

As shown in the Table 2, in case of absolute number of tweets maximum correlation has been found between SLP-Rank and Rank-Abs-U i.e. 0.654 and the least has been found between DO-Rank and Rank-Abs-G i.e. 0.056. From the above analysis, it can be seen that the number of tweets alone cannot be considered enough to evaluate the performance of cities in cleanliness. Thus, different demographic characteristics of the cities have been taken into account to generalize Twitter information. These characteristics have been further used as the features for different prediction algorithms i.e. Linear Regression, Decision Tree Regression, Random Forest Regression and Support Vector Regression for Linear Kernel and RBF kernel. The demographic features used are as follows: Number of Tweets from Public Handles, Number of Tweets from Government Handles, Population, Area in Square Kilometers, Female Population, Percentage decade growth in the population of the city.

3.4.3 Dataset Pre-processing. The dataset pre-processing procedure explained in this section has been performed in addition to the pre-processing of Twitter data performed in Section 3.1.1. The input dataset consists of total nine attributes i.e. Number of Tweets from Public Handles, Number of Tweets from Government Handles, Population, Area in Square Kilometers, Female Population and Percentage decade growth in the population of the city. All the attributes used in this data set such as population, overall scores by government, and area of the city belong to very different scales. Hence, the data has been first normalized using the $z$-score Normalization to convert the attribute with mean value 0 and variance 1 , as given in equation 14 .

$$
z_{i}=\left(x_{i}-\mu\right) / \sigma
$$

where $x$ is attribute value, $\mu$ is the mean of the attribute and $\sigma$ is the standard deviation of the attribute. Further to fill the missing values, we used the forward fill and backward fill on attribute values arranged in ascending order. Forward fill technique fills the empty cell with the previous non-empty cell, where backward fill technique fills an empty cell with the next non-empty cell.

After pre-processing the dataset, regression-based prediction models have been applied to predict the scores of the cities. For the sake of generalization, five different types of prediction models have been applied. Also, as the 
government has provided data for only 4023 cities, the dataset for supervised training is limited only to 4023 instances. However, the model once fitted can be used to predict the scores for other cities. Due to this limitation in data, advanced prediction model such as deep learning fails to fit the model. The government provided scores have been used as the target values for supervised learning of all the prediction models. The training has been done over $70 \%$ of the dataset. The rest of the $30 \%$ dataset has been used for testing the model. The regression models used for the comparison are Multiple Linear Regression [17], Decision Tree Regression [32], Random Forest Regression [19], Support Vector Regression using Linear Kernel, and Support Vector Regression using Radial Basis Function [39].

Multiple Linear Regression. Multiple Linear Regression [17] is the linear approach of predicting the dependent variable given multiple independent variables. Linear regression uses the linear predictor function to estimate the unknown parameters using the observed data set. Given a dataset $\left\{y_{i}, \mathbf{x}_{\mathbf{i}}\right\}$ where $\mathbf{x}_{\mathbf{i}}$ is a $p$-vector independent variable and $y_{i}$ is the dependent variable. Equation 15 gives the prediction for the linear regression for $n$ statistical units and $\varepsilon$ being the error or unobserved noise.

$$
y_{i}=x_{i}^{T} \beta+\varepsilon_{i}, i=1,2, \ldots, n
$$

Decision Tree Regression. Decision tree [32] uses the tree-like structure that contains condition controlled statements to make a decision. These are generally easy to interpret and generate values for even little hard data. The $n$-ary trees have the internal nodes as the tests/conditions and external nodes represent the outputs of these conditions. The internal node is split if the split induces a decrease in impurity greater than or equal to the value as given in Equation 16.

$$
\frac{N_{t}}{N} \cdot\left(\text { impurity }-\frac{N_{t_{R}}}{N_{t}} \cdot \text { right }_{\text {impurity }}-\frac{N_{t_{L}}}{N_{t}} \cdot \text { left }_{\text {impurity }}\right)
$$

where, $N$ is the total number of samples, $N_{t}$ is the number of samples at the current node, $N_{t_{L}}$ is the number of samples in the left child, and $N_{t_{R}}$ is the number of samples in the right child.

The model has been fine-tuned to find an optimal value of some parameters of the decision tree. First, the model has been trained over a range of Tree-Depth values i.e. (1,32). The training and testing errors are calculated as Root Mean Squared Error (RMSE) and has been plotted in the Figure 8 for Tree-Depth values from 1 to 32. According to the figure above, the test set results are minimum, before reaching a saturation, at max_depth $=16$. The other parameters such as min_samples_leaf and min_samples_split have been allowed to vary dynamically.

Random Forest Regression. The random forest regression model [19] is an ensemble learning approach to predict the output. Random forests fit multiple numbers of bootstrapped decision tree classification models to input size sub-samples of the dataset. As the regressor model uses the decision trees for predicting the output, the node splitting and parameter tuning have been kept the same as the decision tree. The random forests help in controlling the generality of the model.

Support Vector Regression using Linear Kernel. The Support Vector Regression model (SVR) [39] uses the similar approach as Support Vector Machines (SVM) [8] where data is fit using a hyperplane while fitting the error into a certain threshold range of the hyperplane. The hyperplane is the best fit line that has the maximum number of points. The first step of SVM regression is converting the input $x$ to an $m$-dimensional feature space using a linear or non-linear mapping (i.e. Kernel). In this work, we have used two types of kernels as given below:

- Linear Kernel: The linear kernel is the simplest mapping function used in SVM. The kernel can be written mathematically as given in Equation 17.

$$
K\left(x, x_{i}\right)=\operatorname{sum}\left(x \cdot x_{i}\right)
$$




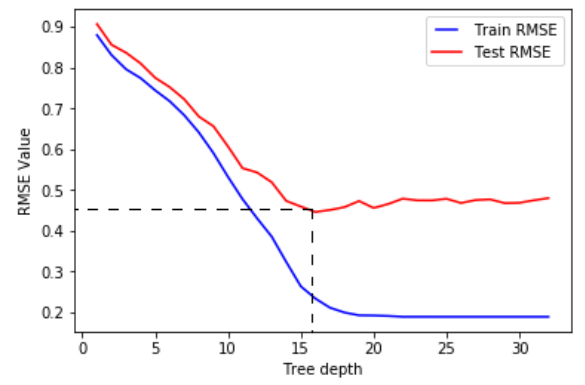

(a) RMSE

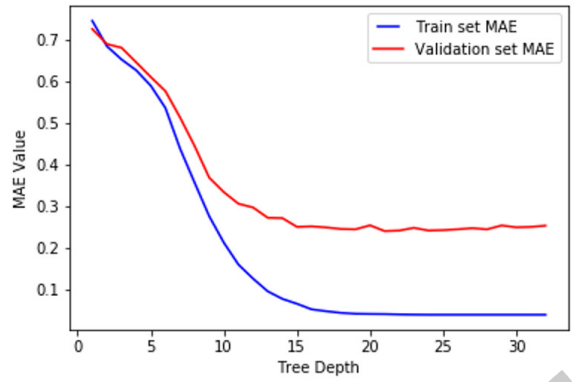

(b) MAE

Fig. 8. RMSE and MAE plot for the range of Tree Depth values for Decision regression

- Radial Basis Function: Radial basis is the complex form of a mapping kernel and can be written mathematically as given in the Equation 18 .

$$
K\left(x, x_{i}\right)=\exp \left(-\gamma \cdot \sum\left(\left(x-x_{i}^{2}\right)\right)\right)
$$

The prediction in SVR is done as given in the Equation 19.

$$
y_{i}=<w, x_{i}>+b \pm \varepsilon
$$

\section{EXPERIMENTATION AND RESULTS}

The performance of the proposed frameworks have been evaluated using the real-time collected Twitter data and the ground truth data collected from the government resources. This section provides the details on the ground truth data and experimentation on the proposed frameworks using the Twitter data.

\subsection{Predicting Citizens' Feedback}

Local Circles [3] is a social media platform which acts as the intermed-iary between the citizens and the government organizations to work towards different issues, challenges, solutions and opportunities on macro and micro levels. A Local Circles online citizen community was initiated by the Ministry of Urban Development of Government of India (GOI) in October 2014 for capturing citizens' feedback for SBA. Since then, over 35,000 citizens have become part of various communities in more than 60 cities in India. In [25], Local Circles performed a national level survey in 2017, where they monitored the impact of SBA after two years of its inception. The objective was to understand the highs and lows of the campaign covering different social and economic aspects of SBA as the different parameters. Broadly, these aspects include topics such as Public Toilet availability, Waste Management, Civic Sense improvement, Municipal Delivery and Responsiveness, Overall Cleanliness Levels of Cities, Impact on Children and Participation by Citizens. They polled more than 115,000 votes in the survey, making it the largest civic survey of its kind. The Local Circles data available online has been used as the ground truth data to validate the proposed framework. On the other hand, our data comprises of 9,00,000 tweets even when there is only $18.9 \%$ of Twitter users in India. Thus, this work has been done on a bigger sample of data as compared to government surveys. Figure 9 depicts the change in the sentiment of the tweets from December 1 to March 31. It shows that most common sentiment is neutral and least common is negative sentiment.

Here, some key highlights of the survey have been analyzed by using Twitter data for the corresponding topics. For the sake of simplicity, we have taken five such topics or highlights from the survey given in [25]. The 


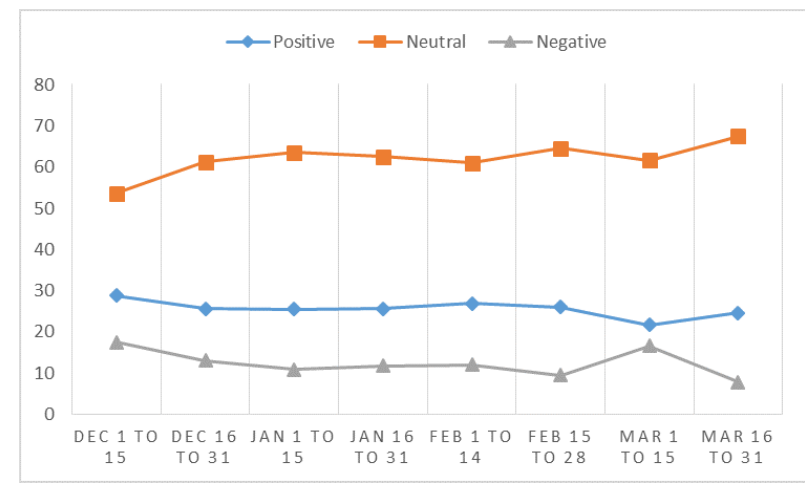

Fig. 9. The change in sentiments of the tweets from December 1 to March 31.

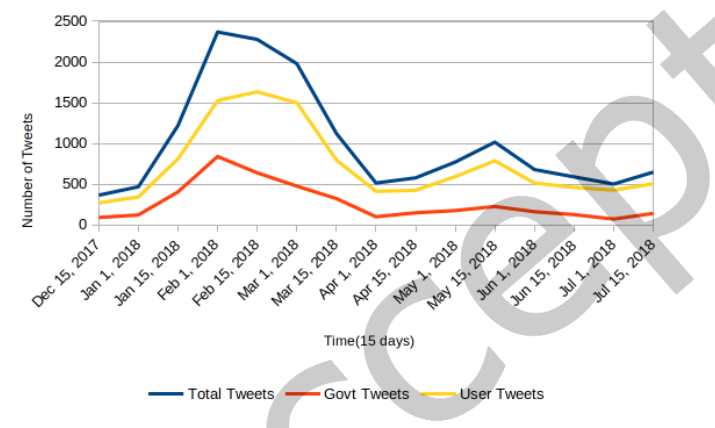

(a) Total number of government and public tweets extracted
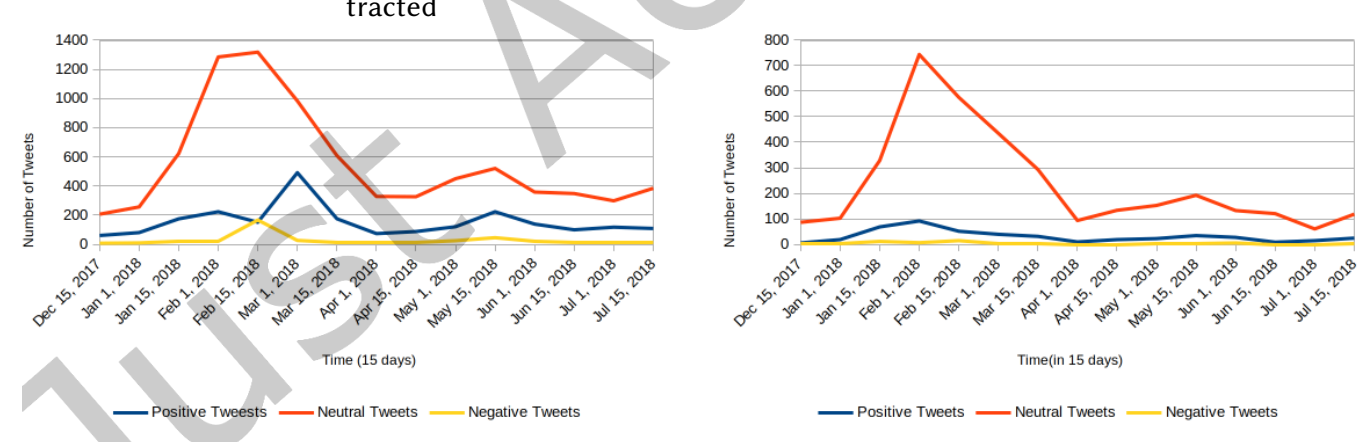

(b) Public generated tweets belonging to different senti-(c) Government generated tweets belonging to different ments sentiments

Fig. 10. The variation in tweet frequencies generated after the processing of tweets related to reviews about the waste disposal management period of December 2017 to July 2018.

highlights taken up here are exemplary and to validate the approach. Other survey topics could also be taken into consideration. However, for the current study, these highlights are thought to suffice the purpose.

The key survey-highlights being used for the validation from the survey are as follows: 

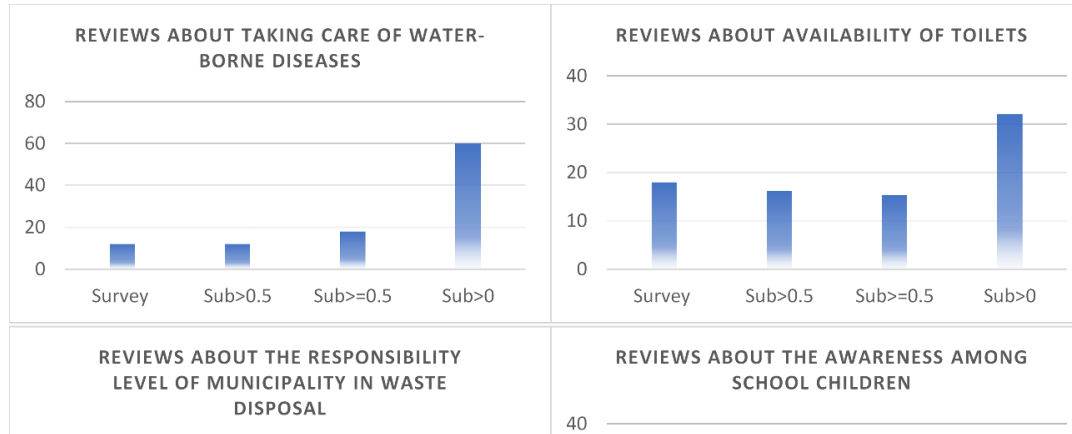

REVIEWS ABOUT THE AWARENESS AMONG DISPOSAL
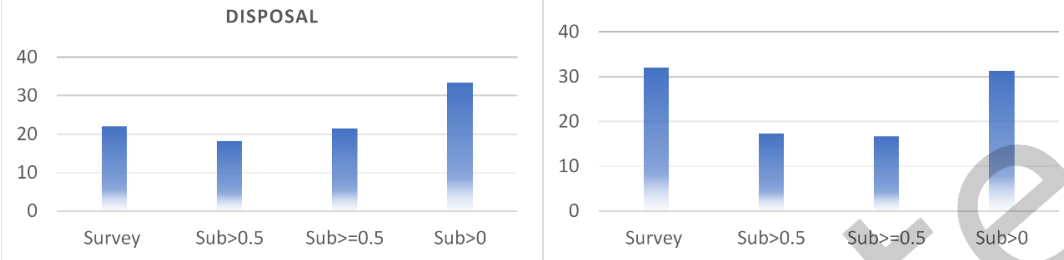

REVIEWS ABOUT MAKING AWARENESS

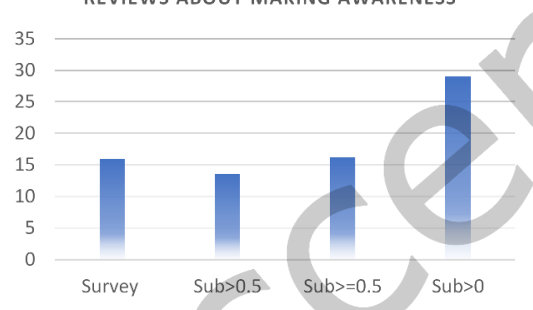

Fig. 11. Bar chart representation of the percentage of tweets found related to a topic under different criteria. Here, sub stands for the subjectivity threshold.

(1) Only $12 \%$ citizens say their municipal corporation is effectively conducting fogging, taking care of water bodies to control vector-borne diseases.

(2) $18 \%$ citizens believe the availability of public toilets in their city has improved since Swachh Bharat.

(3) Only $22 \%$ believe their municipality has become more responsive to complaints on garbage collection, waste disposal, and street cleaning.

(4) $32 \%$ citizens believe Swachh Bharat has been effective in making School Children aware and impart values like cleanliness and civic sense.

(5) Only $16 \%$ citizens believe their municipality is engaged in driving cleanliness and civic sense awareness initiatives on the ground.

Figure 10 presents in the variation in tweet frequencies for the topic "reviews related to waste disposal management" over the period of December 2017 to July 2018. Figure 10(a) gives the total number of tweets, government-generated tweets, and public/user-generated tweets. Figure 10(b) gives the number of positive, negative and neutral tweets posted by the common public. Similarly, Figure 10(c) gives the number of positive, negative and neutral tweets posted by using the government handles. This graph shows that there is a high frequency of tweets in the months from January to March; which is also the time period of government based on-field surveys. Also, the maximum number of tweets generated are neutral as shown by the red lines in $10(\mathrm{~b})$ 
Table 3. The correlation between the survey data results and the Twitter data results

\begin{tabular}{lll}
\hline Sr.No. & Subjectivity score & Correlation Coefficient \\
\hline 1 & Sub $>0.5$ & 0.7771 \\
2 & Sub $\geq 0.5$ & 0.4921 \\
3 & Sub $>0$ & -0.5574 \\
\hline
\end{tabular}

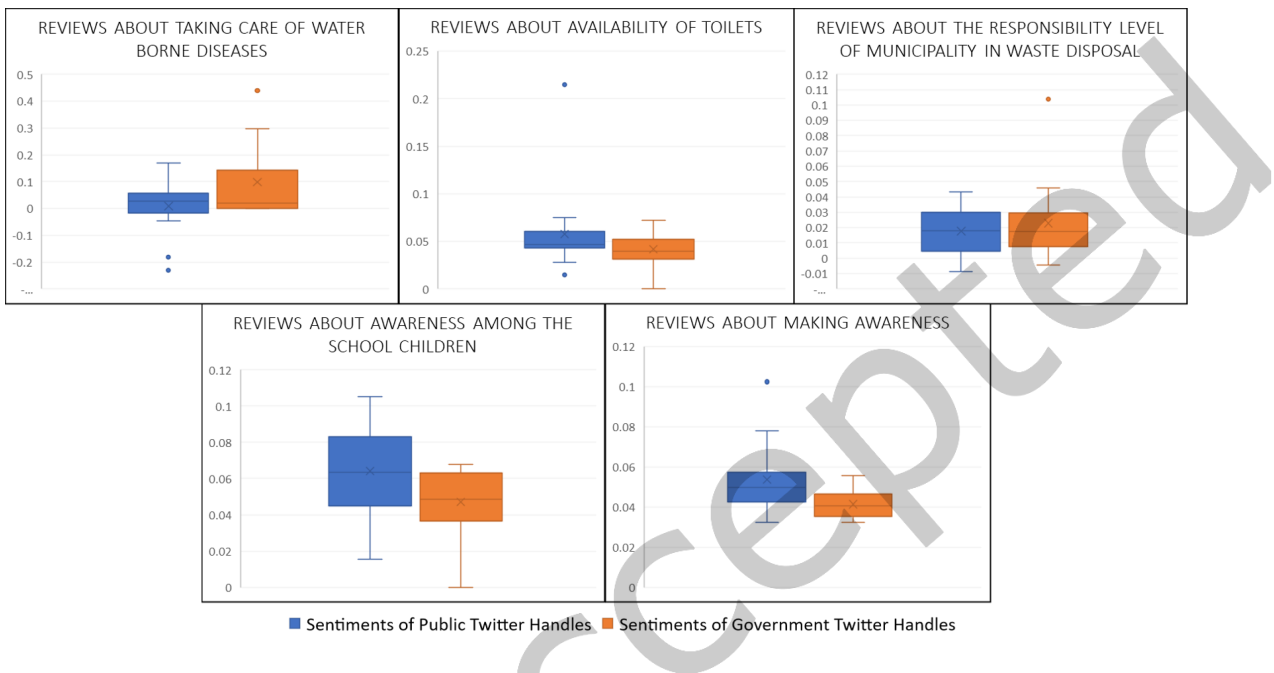

Fig. 12. Box plot representation of the average sentiments of Public and Government handles half-monthly for the different survey topics

and (c). This indicates that there is a very low percentage of tweets that show any emotion and thus most of the tweets can be considered only informative in nature.

Figure 11 presents a bar chart representation of the percentage of tweets showing the respective sentiments as given in the survey highlighted for all the types of subjectivity scores. For example, for survey highlight \#1, 12\% people have shown a positive sentiment towards taking care of vector-borne diseases by taking care of the water bodies. Thus, only the percentage of positive tweets posted by regular users (corresponding to citizens) related to this have been taken into consideration. It can be seen through the chart in Figure 11 that the results of the survey closely match with the sentiments achieved by using Twitter data containing related set of keywords when $S u b>0.5$. However, it can be seen that there is a huge difference between the survey results and the Twitter results with $S u b>0$. The reason behind this could be that these tweets tend to show very less emotional content and using such tweets to collect the sentiments related to a topic could be misleading at times.

Table 3 gives the Pearson's correlation coefficients found between the results of the manual survey and that generated using Twitter data. This table shows that the survey data has the highest correlation with the Twitter data when sentiment scores of the tweets have been calculated with $S u b>0.5$. Similarly, the difference between correlation shown by $S u b>0.5$ and that of $S u b \geq 0.5$ indicates towards the fact that removing the tweets related to $S u b==0.5$ eliminates the tweets showing an ambiguous content. This helps in improving the accuracy of the results. 
Table 4. The results of different predictor models in terms of given evaluation metrics. Here, the bold values represent the best values of the performance metrics achieved.

\begin{tabular}{cccccc}
\hline $\begin{array}{c}\text { Predictor } \\
\text { Model }\end{array}$ & $\begin{array}{c}\text { Explained } \\
\text { Variance } \\
\text { Score }\end{array}$ & $\begin{array}{c}\text { Mean } \\
\text { Absolute } \\
\text { Error }\end{array}$ & $\begin{array}{c}\text { Mean } \\
\text { Square } \\
\text { Error }\end{array}$ & R-Square & Accuracy \\
\hline Linear Regression & 0.2384 & 0.7632 & 0.7860 & 0.2359 & $0.14(+/-0.21)$ \\
Decision Tree Regression & 0.63006 & 0.32521 & 0.38058 & 0.63006 & $0.67(+/-0.38)$ \\
Random Forest Regression & $\mathbf{0 . 8 3 2 8}$ & $\mathbf{0 . 2 6 9 0}$ & $\mathbf{0 . 1 7 2 4 8}$ & $\mathbf{0 . 8 3 2 3}$ & $\mathbf{0 . 8 0}(+/-\mathbf{0 . 1 5})$ \\
SVR (Linear) & 0.2497 & 0.7472 & 0.7952 & 0.22697 & $0.09(+/-0.36)$ \\
SVR (RBF) & 0.3575 & 0.6604 & 0.67139 & 0.3473 & $0.19(+/-0.25)$ \\
\hline
\end{tabular}

The unstructured nature of Twitter data can be used in many other manners to generate more insights which may not be available through the online and offline survey results. Here, we further analyze the Twitter data to view a difference between the public and government generated Twitter sentiments. Here, we divide the whole nine-month data into subsets of 15 days each and plot the average sentiments of each 15 days in the form of box plot as shown in Figure 12. Here, we can see that the average sentiments of the government generated tweets are generally towards zero i.e. more neutral as compared to the Public generated tweets in most of the cases. This analysis shows that government tweets tend to show less emotional or opinionated content than the public generated tweets. However, the topic: 'Taking care of water-borne diseases' can be considered very interesting here because of its anomalous behavior and hence this topic can be studied in more details to find the reasons behind the negative sentiments in public about this topic.

\subsection{Demographic Features based Performance Predictor}

The performance evaluation of the predictor models has been performed using the following performance evaluation metrics where, $\hat{y}$ is the estimated target output, $y$ the corresponding (correct) target output, and Var is Variance, the square of the standard deviation.

Explained Variance Score. Explained variance score measures the proportion to which a mathematical model accounts for the variation (dispersion) of a given data set. If $\hat{y}$ is the estimated target output, $y$ the corresponding (correct) target output, and Var is Variance, the square of the standard deviation, then the explained variance is estimated as given in the Equation 20. The best possible score is 1.0, lower values are worse.

$$
\text { explained_variance }(y, \hat{y})=1-\frac{\operatorname{Var}\{y-\hat{y}\}}{\operatorname{Var}\{y\}}
$$




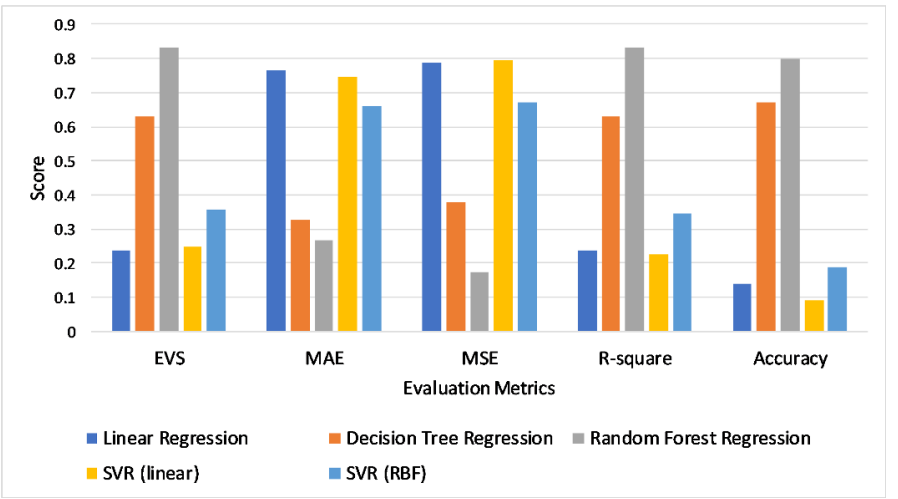

Fig. 13. The bar graph plot representation of the different evaluation metrics for different predictor models 
111:24 • Dhiman and Toshniwal

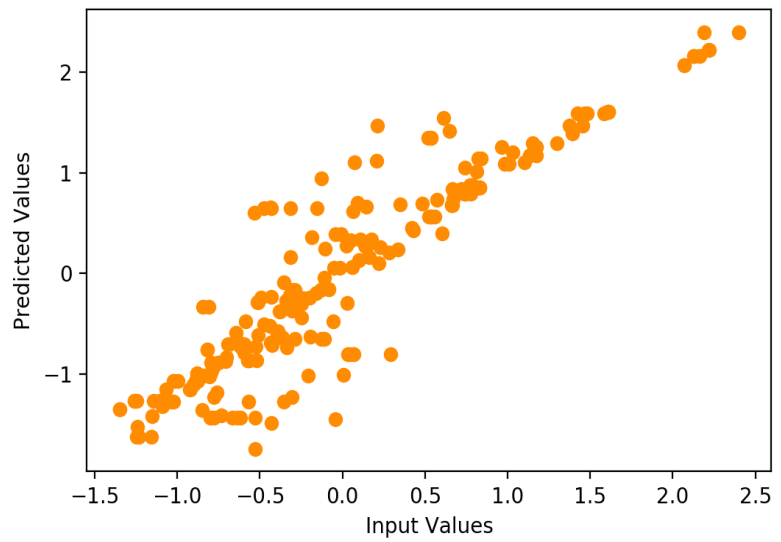

Fig. 14. Scatter Plot of model predictions against the target government scores for Random Forest Regression

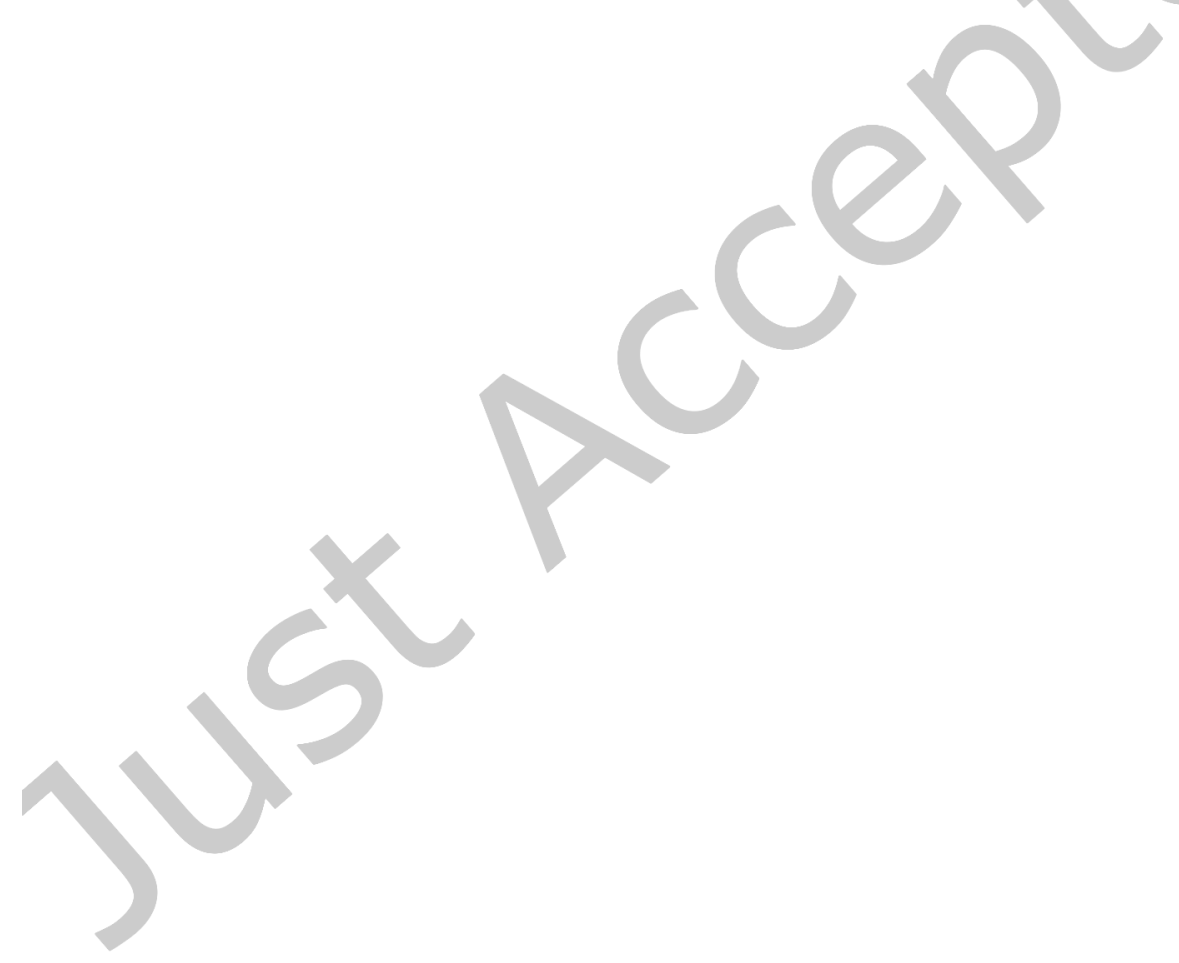


Table 5. Some important characteristics of the resultant clusters

\begin{tabular}{|c|c|c|}
\hline $\begin{array}{l}\text { Cluster } \\
\text { Name }\end{array}$ & $\begin{array}{l}\text { Percentage of } \\
\text { cities in the } \\
\text { cluster }\end{array}$ & $\begin{array}{l}\text { Most Frequent States } \\
\text { in the cluster }\end{array}$ \\
\hline Cluster 1 & 40.446 & $\begin{array}{l}\text { Bihar, Uttar Pradesh, } \\
\text { Tamil Nadu, Gujarat }\end{array}$ \\
\hline Cluster 2 & 54.975 & $\begin{array}{c}\text { Maharashtra, Jharkhand, } \\
\text { Madhya Pradesh, Andhra Pradesh }\end{array}$ \\
\hline Cluster 3 & 0.2487 & Delhi \\
\hline Cluster 4 & 3.7313 & $\begin{array}{l}\text { Maharashtra, Kerala, } \\
\text { Gujarat, Andhra Pradesh }\end{array}$ \\
\hline
\end{tabular}

Mean Absolute Error (MAE). The mean absolute error is a risk metric corresponding to the expected value of the absolute error loss or $l 1-$ norm loss. If $\hat{y}$ is the predicted value of the $i$-th sample, and $y$ is the corresponding true value, then the mean absolute error $(M A E)$ estimated over $n_{\text {samples }}$ is given in the Equation 21 .

$$
\operatorname{MAE}(y, \hat{y})=\frac{1}{n_{\text {samples }}} \sum_{i=0}^{n_{\text {samples }}-1}\left|y_{i}-\hat{y}_{i}\right|
$$

Mean Squared Error(MSE). Mean Square Error is a risk metric corresponding to the expected value of the squared (quadratic) error or loss. If $\hat{y}$ is the predicted value of the $i$-th sample, and $y$ is the corresponding true value, then the mean squared error $(M S E)$ estimated over $n_{\text {samples }}$ is given in the Equation 22.

$$
\operatorname{MSE}(y, \hat{y})=\frac{1}{n_{\text {samples }}} \sum_{i=0}^{n_{\text {samples }}-1}\left(y_{i}-\hat{y}_{i}\right)^{2}
$$

$R$-Square $\left(R^{2}\right)$. R-Square score i.e. the coefficient of determination provides a measure of how well future samples are likely to be predicted by the model. If $\hat{y}$ is the predicted value of the $i$-th sample and $y$ is the corresponding true value, then the score $R^{2}$ estimated over $n_{\text {samples }}$ is given in the Equation 23 .

where, $\bar{y}=\frac{1}{n_{\text {samples }}} \sum_{n_{\text {samples }}-1}^{i=0} y_{i}$

$$
R^{2}(y, \hat{y})=1-\frac{\sum_{i=0}^{n_{\text {samples }^{-1}}\left(y_{i}-\hat{y}_{i}\right)^{2}}}{\sum_{i=0}^{n_{\text {samples }}-1}\left(y_{i}-\overline{y_{i}}\right)^{2}},
$$

Accuracy. Accuracy is defined as the proportion of correct predictions in the total predictions as given in Equation 24. Here, accuracy has been calculated as a measure of cross-validation. $k$-fold cross-validation a resampling technique that divides the data in $\mathrm{k}$ parts, where training is done on $k-1$ parts and testing is done on the remaining part using the hold-out method. We use the mean score and the $95 \%$ confidence interval of the score estimated for 10 -fold cross-validation on the dataset.

$$
\text { Accuracy }=\frac{\text { correctprediction }}{\text { allpredictions }}
$$

All the models are applied to the test set to predict the performance of the cities and the corresponding results of evaluation metrics as given above have been calculated. The individual error metric evaluations have been 
given in Table 4 and Figure 13 for all the models. Table 4 indicates that Random Forest is the best predictor with accuracy $0.80(+/-0.15)$ followed by Decision Tree Regression with accuracy $0.67(+/-0.38)$ for the given dataset. Figure 14 gives a scatter plot of the predicted values against the target values for Random Forest Regression. It is visible from the plot that the predictions have a high correlation with the target values. This implicates the usefulness of Twitter in evaluating the performance of the campaign per city. The traditional methods of performing this task are already very labor, cost and time intensive approaches.

4.2.1 Clustering Cities to find Interesting Patterns. Further, the cities have been clustered using Twitter and the demographic information as the features of K-Means Clustering. This analysis helps to determine some interesting patterns among the behavior of the cities. This data set consists of the total six features i.e., Government Scores, Predicted Twitter Scores, Area in square Kilometer, Population, Female population, Decade growth in the population. Table 5 provides the percentage of cities in each cluster and the states which have the maximum number of its cities in each cluster. From Table 5 it can be seen that Cluster 1 and 2 are the most populated and Cluster 3 is the most sparse of all.

Interestingly, cluster 3 only contains one place that is, Delhi. The reason behind this interesting behavior shown by Delhi, being the National Capital of India, could be a very high population, small area and very high number of tweets generated for this place. Also, Cluster 4 consists of cities such as Indore, Pune, Nagpur, Mumbai, etc.

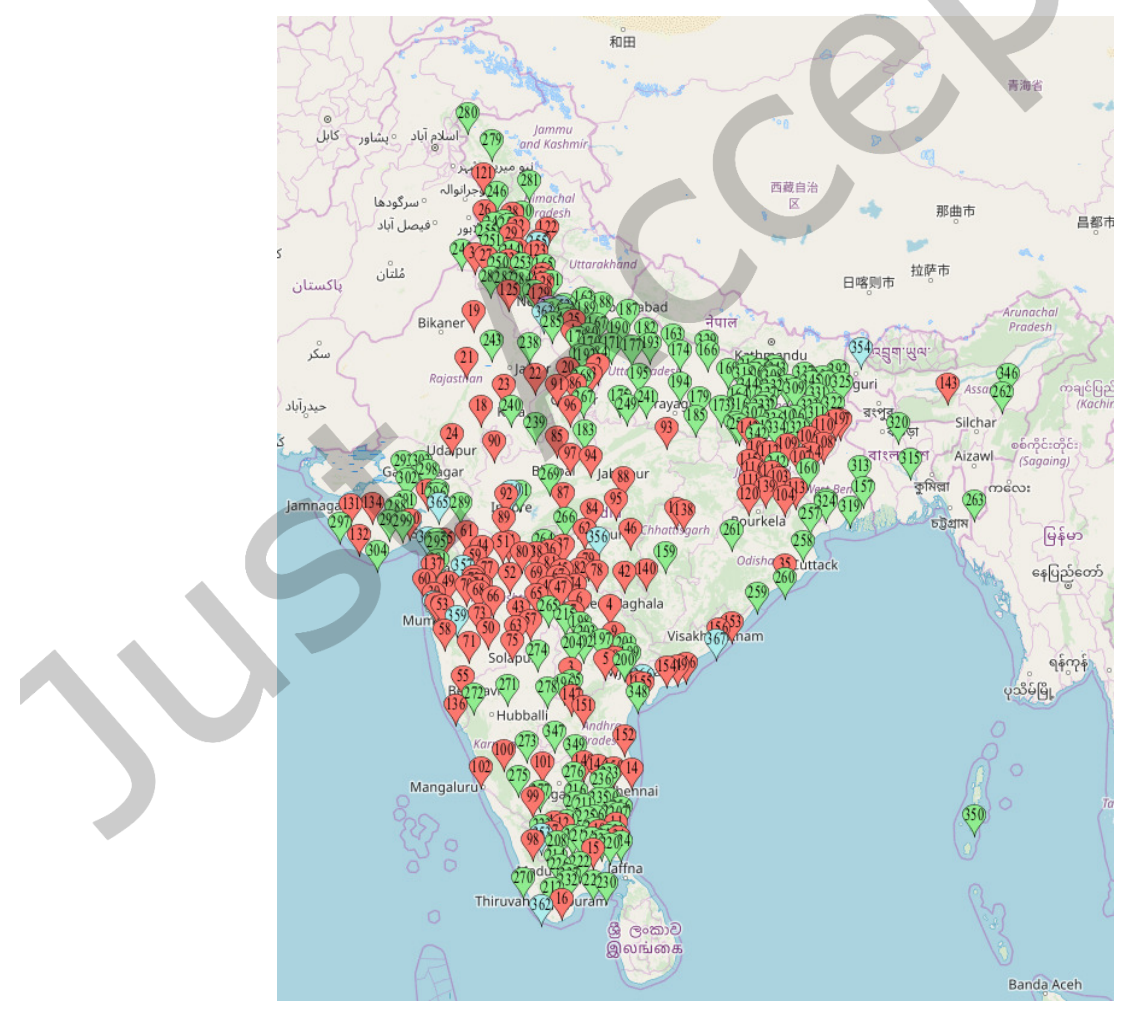

Fig. 15. Classifying whole data into four classes on Government and Predicted Scores using all the demographic characteristics of the cities 
Though Cluster 4 contains a very small number of cities, these are the cities which have been ranked at the top according to the Swachh Survekshan 2018 rankings given by the government.

From Table 5, Maharashtra can be stated as showing the most interesting behavior in the country, as the cities in this state are most frequently occurring in these clusters. According to Swachh Survekshan 2018, Maharashtra has been ranked second in best performing states in the country. Other states such as Jharkhand, Andhra Pradesh, Madhya Pradesh have been ranked in top 5 according to the government criteria which are also visible in the above table as the states with the highest number of corresponding cities. Similarly, the states such as Uttar Pradesh, Tamil Nadu, Gujarat, Kerala have been ranked between 11 and 20 by the government standards. However, the states such as Telangana, Sikkim, Manipur, etc., which have been ranked in the top 10 by the government standards could not be seen as much active on Twitter. Thus, this analysis helps in finding out the cities as well as states which are showing an interesting behavior.

Figure 15 shows the scatter plot of the clusters on the map of India. From Figure 15, it can be seen that Cluster 1 is prominent in the North Zone of India and Cluster 2 is prominent in the Center Zone of India. However, there is a very small number of points for cluster 3 and 4 and thus these are not apparently visible on the map.

\section{CONCLUSION}

In this research work, Twitter data related to Indian Cleanliness Campaign; Swachh Bharat Abhiyan (SBA) has been processed to present the application of Twitter data in evaluating the effectiveness of a government campaign. The primary contribution of this work in assessing the effectiveness is using the Twitter data to reproduce the results that are otherwise provided by some manual surveys. The surveys can be of two types i.e. one related to citizen feedback; which can be easily reproduced using the tweet pooling based sentiment analysis framework and second is doing the field and paper-based suryeys; which were reproduced by using the demographic features based prediction model on the Twitter data. This work achieved a 0.77 correlation in modeling the perceptions of Indian citizens towards the impact of cleanliness and an $80(+/-15) \%$ accuracy in generalizing the Twitter data to predict the performance of different cities in maintaining cleanliness. This analysis shows that just like many other tasks, Twitter is very well suited to reproduce the information generated by the online/offline surveys. Thus, this work addresses the issue of understanding public responses to the governmental campaigns for developing countries such as India as well.

Further, an application of Twitter data in comparing the cities using the clustering analysis has been presented. The cluster analysis of the Twitter data rankings gave us the names of the cities and states showing some interesting patterns. For example, Delhi is the only place which has occurred alone in the cluster due to its very special combination of demographic characteristics. Overall, the interesting behaviors of the cluster analysis of the cities and their corresponding states were clearly validated by the fact that these cities and states have also received recognition in the government surveys. In future work, a combination of other online sources such as Google Trends and news data can be utilized to generate more accurate predictor model with more functionality. Also, these models can be extended further to assess the affects of such government campaigns on the smart cities.

\section{REFERENCES}

[1] 2019. Global Digital Report 2019. https://wearesocial.com/global-digital-report-2019

[2] 2019. National Portal of India. https://www.india.gov.in/my-government/documents/policy

[3] 2019. Social Media for Communities, Local Social Media Network. https://www.localcircles.com/a/

[4] Eiji Aramaki, Sachiko Maskawa, and Mizuki Morita. 2011. Twitter catches the flu: detecting influenza epidemics using Twitter. In Proceedings of the conference on empirical methods in natural language processing. Association for Computational Linguistics, $1568-1576$.

[5] John W Ayers, J Lee Westmaas, Eric C Leas, Adrian Benton, Yunqi Chen, Mark Dredze, and Benjamin M Althouse. 2016. Leveraging big data to improve health awareness campaigns: a novel evaluation of the Great American Smokeout. FMIR public health and surveillance 2 , 1 (2016). https://doi.org/10.2196/publichealth.5304 
[6] Stefano Baccianella, Andrea Esuli, and Fabrizio Sebastiani. 2010. Sentiwordnet 3.0: an enhanced lexical resource for sentiment analysis and opinion mining.. In LREC, Vol. 10. 2200-2204.

[7] Olfa Belkahla Driss, Sehl Mellouli, and Zeineb Trabelsi. 2019. From citizens to government policy-makers: Social media data analysis. Government Information Quarterly (May 2019). https://doi.org/10.1016/j.giq.2019.05.002

[8] Olivier Chapelle and Vladimir Vapnik. 2000. Model selection for support vector machines. In Advances in neural information processing systems. $230-236$.

[9] Cynthia Chew and Gunther Eysenbach. 2010. Pandemics in the age of Twitter: content analysis of Tweets during the 2009 H1N1 outbreak. PloS one 5, 11 (2010), e14118. https://doi.org/10.1371/journal.pone.0014118

[10] citypop 2018. Population Statistics for Countries, Administrative Areas, Cities and Agglomerations. https://www.citypopulation.de/ India.html

[11] Qi Dang, Feng Gao, and Yadong Zhou. 2016. Early detection method for emerging topics based on dynamic bayesian networks in micro-blogging networks. Expert Systems with Applications 57 (2016), 285-295. https://doi.org/10.1016/j.eswa.2016.03.050

[12] Munmun De Choudhury, Michael Gamon, Scott Counts, and Eric Horvitz. 2013. Predicting depression via social media. ICWSM 13 (2013), 1-10.

[13] Nic DePaula, Ersin Dincelli, and Teresa M. Harrison. 2018. Toward a typology of government social media communication: Democratic goals, symbolic acts and self-presentation. Government Information Quarterly 35, 1 (Jan. 2018), 98-108. https://doi.org/10.1016/j.giq 2017.10.003

[14] Aarzoo Dhiman and Durga Toshniwal. 2018. Do Public and Government Think Similar About Indian Cleanliness Campaign?. In Annual International Symposium on Information Management and Big Data. Springer, 367-380. https://doi.org/10.1007/978-3-030-11680-4_34

[15] Saptarshi Dutta. 2017. How Are Swachh Bharat Abhiyan And Namami Gange Benefitting From Other-Countries? http://swachhindia. ndtv.com/how-are-swachh-bharat-abhiyan-and-namami-gange-benefitting-from-othercountries-8684/.htm

[16] Seok-Jin Eom, Hanchan Hwang, and Jun Houng Kim. 2018. Can social media increase government responsiveness? A case study of Seoul, Korea. Government Information Quarterly 35, 1 (Jan. 2018), 109-122. https://doi.org/10.1016/j.giq.2017.10.002

[17] David A Freedman. 2009. Statistical models: theory and practice. cambridge university press.

[18] Jeremy Ginsberg, Matthew H Mohebbi, Rajan S Patel, Lynnette Brammer, Mark S Smolinski, and Larry Brilliant. 2009. Detecting influenza epidemics using search engine query data. Nature 457, 7232 (2009), 1012. https://doi.org/10.1038/nature07634

[19] Tin Kam Ho. 1995. Random decision forests. In Proceedings of 3rd international conference on document analysis and recognition, Vol. 1. IEEE, 278-282.

[20] Heather A Johnson, Michael M Wagner, William R Hogan, Wendy W Chapman, Robert T Olszewski, John N Dowling, Gary Barnas, et al. 2004. Analysis of Web access logs for surveillance of influenza.. In Medinfo. 1202-1206.

[21] Hian Chye Koh, Gerald Tan, et al. 2011. Data mining applications in healthcare. Fournal of healthcare information management 19, 2 (2011), 65. https://doi.org/10.4314/ijonas.v5i1.49926

[22] Vasileios Lampos and Nello Cristianini. 2010. Tracking the flu pandemic by monitoring the social web. In Cognitive Information Processing (CIP), 2010 2nd International Workshop on. IEEE, 411-416. https://doi.org/10.1109/cip.2010.5604088

[23] Vasileios Lampos, Tijl De Bie, and Nello Cristianini. 2010. Flu detector-tracking epidemics on Twitter. In foint European conference on machine learning and knowledge discovery in databases. Springer, 599-602. https://doi.org/10.1007/978-3-642-15939-8_42

[24] Dana M Litt, Melissa A Lewis, Emma S Spiro, Lovenoor Aulck, Katja A Waldron, Maya K Head-Corliss, and Alex Swanson. 2018. \# drunktwitter: Examining the relations between alcohol-related Twitter content and alcohol willingness and use among underage young adults. Drug and alcohol dependence 193 (2018), 75-82.

[25] localcircle 2018. Local Circles: Citizen assessment of 3 years of Swachh Bharat. https://www.localcircles.com/a/press/page/ 3yearscitizenassessmentswachhbharat\#.W818XRThW90

[26] Baojun Ma, Nan Zhang, Guannan Liu, Liangqiang Li, and Hua Yuan. 2016. Semantic search for public opinions on urban affairs: A probabilistic topic modeling-based approach. Information Processing \& Management 52, 3 (May 2016), 430-445. https://doi.org/10.1016/j. ipm.2015.10.004

[27] Maimuna S Majumder, Mauricio Santillana, Sumiko R Mekaru, Denise P McGinnis, Kamran Khan, and John S Brownstein. 2016. Utilizing nontraditional data sources for near real-time estimation of transmission dynamics during the 2015-2016 Colombian Zika virus disease outbreak. FMIR public health and surveillance 2, 1 (2016). https://doi.org/10.2196/publichealth.5814

[28] Tomas Mikolov, Kai Chen, Greg Corrado, and Jeffrey Dean. 2013. Efficient estimation of word representations in vector space. arXiv preprint arXiv:1301.3781 (2013).

[29] Marwa Naili, Anja Habacha Chaibi, and Henda Hajjami Ben Ghezala. 2017. Comparative study of word embedding methods in topic segmentation. Procedia computer science 112 (2017), 340-349.

[30] Philip M Polgreen, Yiling Chen, David M Pennock, Forrest D Nelson, and Robert A Weinstein. 2008. Using internet searches for influenza surveillance. Clinical infectious diseases 47, 11 (2008), 1443-1448. https://doi.org/10.1086/593098

[31] Shengsheng Qian, Tianzhu Zhang, Changsheng Xu, and M. Shamim Hossain. 2015. Social Event Classification via Boosted Multimodal Supervised Latent Dirichlet Allocation. ACM Trans. Multimedia Comput. Commun. Appl. 11, 2 (Jan. 2015), 27:1-27:22. https://doi.org/10. 
$1145 / 2659521$

[32] J. Ross Quinlan. 1987. Simplifying decision trees. International journal of man-machine studies 27, 3 (1987), 221-234.

[33] Sahil Raj and Tanveer Kajla. 2015. Sentiment analysis of Swachh Bharat Abhiyan. International fournal of Business Analytics and Intelligence 3, 1 (2015), 32. https://doi.org/10.21863/ijbai/2015.3.1.005

[34] Christopher G. Reddick, Akemi Takeoka Chatfield, and Adegboyega Ojo. 2017. A social media text analytics framework for double-loop learning for citizen-centric public services: A case study of a local government Facebook use. Government Information Quarterly 34, 1 (Jan. 2017), 110-125. https://doi.org/10.1016/j.giq.2016.11.001

[35] SBM2018 2018. Swachh Bharat Mission- Gramin. http://swachhbharatmission.gov.in/sbmcms/index.htm

[36] Daniel Scanfeld, Vanessa Scanfeld, and Elaine L Larson. 2010. Dissemination of health information through social networks: Twitter and antibiotics. American journal of infection control 38, 3 (2010), 182-188. https://doi.org/10.1016/j.ajic.2009.11.004

[37] Philip Sedgwick. 2012. PearsonâĂŹs correlation coefficient. Bmj 345 (2012).

[38] Minesh P Shah, Benjamin A Lopman, Jacqueline E Tate, John Harris, Marcelino Esparza-Aguilar, Edgar Sanchez-Uribe, Vesta Richardson, Claudia A Steiner, and Umesh D Parashar. 2017. Use of Internet search data to monitor rotavirus vaccine impact in the United States, United Kingdom, and Mexico. Journal of the Pediatric Infectious Diseases Society (2017), pix004. https:/doi.org/10.1093/jpids/pix004

[39] Alex J Smola and Bernhard Schölkopf. 2004. A tutorial on support vector regression. Statistics and computing 14, 3 (2004), 199-222.

[40] SwachhSurvek2018 2018. Swachh Survekshan 2018. https://www.swachhsurvekshan2018.org

[41] Devendra K Tayal and Sumit K Yadav. 2017. Sentiment analysis on social campaign âĂIJSwachh Bharat AbhiyanâĂİ using unigram method. AI \& SOCIETY 32, 4 (2017), 633-645. https://doi.org/10.1007/s00146-016-0672-5

[42] Matthew S Thiese. 2014. Observational and interventional study design types; an overview. Biochemia medica: Biochemia medica 24, 2 (2014), 199-210. https://doi.org/10.11613/bm.2014.022

[43] Tony. 2019. Social Media in Government: Benefits, Challenges, and How it's Used. https://blog.hootsuite.com/social-media-government/

[44] Laurens Van der Maaten and Geoffrey Hinton. 2008. Visualizing data using t-SNE. fournal of machine learning research 9, 11 (2008). 